ARTICLE

https://doi.org/10.1038/s41467-019-11839-w

\title{
Ubiquitination of RIPK1 suppresses programmed cell death by regulating RIPK1 kinase activation during embryogenesis
}

Xixi Zhang ${ }^{1,5}$, Haiwei Zhang ${ }^{1,5}$, Chengxian $\mathrm{Xu}^{1}$, Xiaoming $\mathrm{Li}^{1}$, Ming $\mathrm{Li}^{1}$, Xiaoxia $\mathrm{Wu}^{1}$, Wenjuan Pu (i) ${ }^{2}$, Bin Zhou (1) ${ }^{2}$, Haikun Wang ${ }^{3}$, Dali Li ${ }^{4}$, Qiurong Ding ${ }^{1}{ }^{1}$, Hao Ying ${ }^{1}$, Hui Wang ${ }^{1} \&$ Haibing Zhang $\mathbb{B}^{1}$

The ubiquitination status of RIPK1 is considered to be critical for cell fate determination. However, the in vivo role for RIPK1 ubiquitination remains undefined. Here we show that mice expressing RIPK1K376R which is defective in RIPK1 ubiquitination die during embryogenesis. This lethality is fully rescued by concomitant deletion of Fadd and Ripk3 or MIkl. Mechanistically, cells expressing RIPK1 ${ }^{\text {K376R }}$ are more susceptible to TNF- $\alpha$ induced apoptosis and necroptosis with more complex II formation and increased RIPK1 activation, which is consistent with the observation that Ripk $7^{K 376 R / K 376 R}$ lethality is effectively prevented by treatment of RIPK1 kinase inhibitor and is rescued by deletion of Tnfr1. However, Tnfr1-/Ripk7 ${ }^{K 376 R / K 376 R}$ mice display systemic inflammation and die within 2 weeks. Significantly, this lethal inflammation is rescued by deletion of Ripk3. Taken together, these findings reveal a critical role of Lys376-mediated ubiquitination of RIPK1 in suppressing RIPK1 kinase activity-dependent lethal pathways during embryogenesis and RIPK3-dependent inflammation postnatally.

\footnotetext{
${ }^{1}$ CAS Key Laboratory of Nutrition, Metabolism and Food Safety, Shanghai Institute of Nutrition and Health, Shanghai Institutes for Biological Sciences, University of Chinese Academy of Sciences, Chinese Academy of Sciences, 100864 Shanghai, China. ${ }^{2}$ The State Key Laboratory of Cell Biology, CAS Center for Excellence on Molecular Cell Science, Shanghai Institute of Biochemistry and Cell Biology, University of Chinese Academy of Sciences, Chinese Academy of Sciences, 100864 Shanghai, China. ${ }^{3}$ Key Laboratory of Molecular Virology and Immunology, Unit of the Regulation of Immune Cell Differentiation, Institute Pasteur of Shanghai, Shanghai Institutes for Biological Sciences, Chinese Academy of Sciences, 100864 Shanghai, China. ${ }^{4}$ Shanghai Key Laboratory of Regulatory Biology, Institute of Biomedical Sciences and School of Life Sciences, East China Normal University (ECNU), 200062 Shanghai, China. ${ }^{5}$ These authors contributed equally: Xixi Zhang, Haiwei Zhang. Correspondence and requests for materials should be addressed to H.Z. (email: hbzhang@sibs.ac.cn)
} 
IPK1, an essential signaling node in various innate immune signaling pathways, is most extensively studied in the TNFR1 signaling pathway. Ligation of TNFR1 results in the rapid formation of a signaling complex termed complex I or TNFR1-Siganaling complex (TNFR1-SC) which is composed of TRADD, RIPK1, TRAF2, and cIAP1/2 ${ }^{1,2}$. Following recruitment, RIPK1 is polyubiquitinated by multiple forms of ubiquitin chains which provides docking sites for two protein complexes, the TAB/ TAK complex comprising TAK1 and TAB2/3, as well as the IKK complex comprising NEMO, IKKa, and IKK $\beta^{3-6}$. The individual formation of these two complexes leads to activation of NF- $\kappa B$ and MAPK signaling pathways and therefore promotes cell survival. Nevertheless, under certain circumstances when the prosurvival signal emanating from complex I is perturbed, RIPK1 dissociates from complex I and recruits FADD and Caspase- 8 to form complex IIa, and this leads to initiation of apoptosis ${ }^{1,7-11}$. However, when apoptosis is inhibited, RIPK1 can interact with RIPK3 to form an amyloid-like complex termed complex IIb or necrosome, resulting in activation of necroptosis ${ }^{12-17}$.

Recently, by extensive genetic studies on RIPK1 signaling pathways, the in vivo role of RIPK1 in regulating both cell survival and cell death has been revealed. Genetic deletion of Ripk1 in animals leads to postnatal lethality with widespread cell death in lymphoid and adipose lineages ${ }^{18}$. Ablation of Caspase-8/Fadd and Ripk3 allows for normal development and maturation of Ripk1-deficient mice ${ }^{19-22}$. Similarly, conditional deletion of Ripk1 in intestinal epithelial cells (IECs) results in premature death in mice accompanied by extensive apoptosis in intestine and ensuing inflammation ${ }^{23,24}$. These phenotypes are largely resolved in mice lacking intestinal Fadd/Caspase- 8 or both Fadd and Ripk $3^{23,24}$. In addition, the mice with keratinocyte-specific Ripk1 deficiency progressively develop severe inflammatory skin lesions that are fully prevented by deletion of Ripk3 or $M l k l^{24}$. Therefore, RIPK1 seems to be a suppressor of lethal pathways engaged by FADD/Caspase- 8 and RIPK3/MLKL. However, RIPK1 is also suggested to mediate cell death in some contexts. Ablation of Ripk1 prevents early embryonic lethality induced by Caspase- 8 or Fadd, although these mice succumb to early postnatal lethality similar to Ripk1 deficient mice $21,22,25$. Another striking study showed that mice with homozygous Ripk $3^{D 161 N}$ died at E10.5 but were completely rescued by co-deletion of Ripk $1^{26}$. These investigations revealed an opposite function of RIPK1 which can also induce cell death. Therefore, the paradoxical roles of RIPK1 raise the following fundamental question: what is the switch for two opposing functions of RIPK1?

RIPK1 consists of an N-terminal serine/threonine kinase domain, an intermediate domain, and a C-terminal death domain (DD) $)^{27,28}$. The kinase activity is essential for induction of necroptosis and RIPK1-dependent apoptosis but is dispensable for NF- $\kappa B$ activation ${ }^{29-31}$. Animals with kinase-inactive mutant RIPK1 develop normally and are protected from TNF- $\alpha$ induced necroptosis in vitro and in vivo, suggesting that the pro-survival function of RIPK1 does not require RIPK1 kinase activity ${ }^{32,33}$. The intermediate domain contains a RIP homotypic interaction motif (RHIM) which binds the RHIM of RIPK3 to initiate necroptosis, whereas the genetic studies reveal a critical role of RHIM in inhibiting ZBP1/RIPK3/MLKL-dependent necroptosis during development and inflammation ${ }^{34-36}$. In addition, a recent work has shown that DD-mediated RIPK1 dimerization is crucial for RIPK1 activation, and mice with mutant DD are resistant to RIPK1-dependent apoptosis and necroptosis ${ }^{37}$. Thus, although these genetic studies have demonstrated that RIPK1 is involved in mediating apoptosis, necroptosis and cell survival, the mechanism by which RIPK1 regulates these pathways in vivo remains unclear.
Given that the ubiquitination status of RIPK1 is critical for the transition of complex I to complex II, a clue to the answer may come from a major functional ubiquitination site K377 of human RIPK1, which provides a docking site for the K-63 linked polyubiquitin chain and mediates the recruitment of TAK1 and IKK complexes ${ }^{3,5,38,39}$. In vitro studies have shown that substitution of Lys-377 with arginine (K377R) blocked RIPK1 ubiquitination and TNF- $\alpha$-mediated NF- $\kappa B$ activation and sensitized cells to TNF- $\alpha$ induced apoptosis $3,5,38,39$. However, the in vivo function of K377, which is the key to uncover the enigma of the paradoxical roles of RIPK1, has not been established.

In this study, we generate mice endogenously expressing RIPK1 ${ }^{\mathrm{K} 376 \mathrm{R}}$, a single amino acid change in K376 of mouse RIPK1, which is homologous to K377 of human RIPK1. Strikingly, mice bearing Ripk $1^{K 376 R / K 376 R}$ die at embryonic day 12.5 (E12.5) with excessive cell death in embryonic tissues and the yolk sac. Accordingly, Mouse embryonic fibroblasts (MEFs) expressing RIPK1 ${ }^{\mathrm{K} 376 \mathrm{R}}$ are defective in TNF- $\alpha$-induced ubiquitination and are more sensitive to TNF- $\alpha$-induced apoptosis and necroptosis. The excessive cell death in mutant embryos which can be effectively prevented by $\mathrm{Nec}-1$ treatment is proved to be dependent on the kinase activity of RIPK1. Intriguingly, Ripk $1^{K 376 R /-}$ mice with only half amounts of mutant RIPK $1^{\mathrm{K} 376 \mathrm{R}}$ are viable although these mice develop systemic inflammation after birth. Besides, ablation of Fadd and Ripk3/Mlkl rescues Ripk $1^{K 376 R / K 376 R}$ mice from embryonic lethality and allows the animals to grow into fertile adults, indicating that the lethal phenotypes of mutant mice are caused by FADD-dependent apoptosis and RIPK3/MLKL dependent necroptosis. Furthermore, deletion of $T n f r 1$ rescues Ripk $1^{K 376 R / K 376 R}$ mice at the embryonic stage but fails to prevent the postnatal systemic inflammation of the mutant mice. Importantly, Ripk3 deficiency prevents lethal inflammation of Tnfr1 ${ }^{-/-}$Ripk $1^{K 376 R / K 376 R}$ mice, suggesting that ubiquitination of RIPK1 is also involved in regulating inflammation during postnatal development. Thus, our findings provide genetic evidences that Lys376-mediated ubiquitination of RIPK1 plays critical roles in regulating both embryogenesis and inflammation processes.

\section{Results}

Ripk1 ${ }^{K 376 R / K 376 R}$ mice die during embryogenesis. To address the potential role of RIPK1 ubiquitination in vivo, we generated knock-in mice with Lysine on a key ubiquitination site mutated to Arginine (K376R) (Fig. 1a). Unexpectedly, unlike Ripk1 $1^{-/-}$mice that died within 3 days after birth, Ripk $1^{K 376 R / K 376 R}$ mice died during embryogenesis as intercrossing of heterozygous mice only generated heterozygous and wild-type (WT) offspring (Fig. 1b). Ripk $1^{K 376 R /+}$ mice had the same normal life span as WT littermates, excluding the possibility that RIPK1K376R acted as a dominant negative mutant. To gain more insight into the lethality of $R i p k 1^{K 376 R / K 376 R}$ mice, we performed timed pregnancies by mating heterozygous animals. The results showed that Ripk $1^{K 376 R / K 376 R}$ embryos and their yolk sacs appeared normal at E11.5 (Fig. 1c). However, staining for TUNEL revealed increasing dead cells in fetal livers of the mutant embryos (Fig. 1d). At E12.5, although the appearances of $R i p k 1^{K 376 R / K 376 R}$ embryos were normal, histological examination showed remarkable tissue losses in parts of fetal livers (Fig. 1c, d). Immunoblot analysis showed activated caspase- 3 and the cleavage of PARP, as well as aggregations of RIPK1 and RIPK3 were clearly detected in body tissues of mutant embryos, suggesting that activation of apoptosis and necroptosis contributes to the cell death in mutant embryos (Fig. 1f). Besides, immunostaining of yolk sacs for VE-cadherin revealed obvious vascular abnormalities with remarkably enhanced caspase- 3 activation in the yolk sacs of mutant 
a

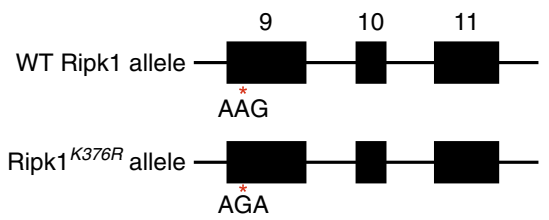

K376

A G A

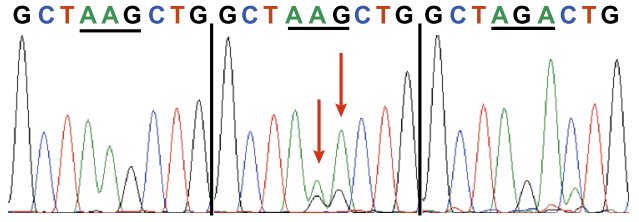

b

\begin{tabular}{|c|c|c|c|c|}
\hline \multicolumn{5}{|c|}{ Ripk1 ${ }^{\mathrm{K} 376 \mathrm{R} /+}$ intercrossing } \\
\hline Stage & Ripk1 ${ }^{+/+}$ & Ripk1 ${ }^{\text {K376R/+ }}$ & Ripk1 $1^{\text {K376R/K376R }}$ & Total \\
\hline E11.5 & 15 & 29 & 10 & 54 \\
\hline E12.5 & 18 & 40 & 16 & 74 \\
\hline E13.5 & 20 & 49 & $19^{*}$ & 88 \\
\hline$>\mathrm{PO}$ & 59 & 77 & 0 & 136 \\
\hline
\end{tabular}

C

E10.5

E11.5

E12.5

E13.5

E14.5
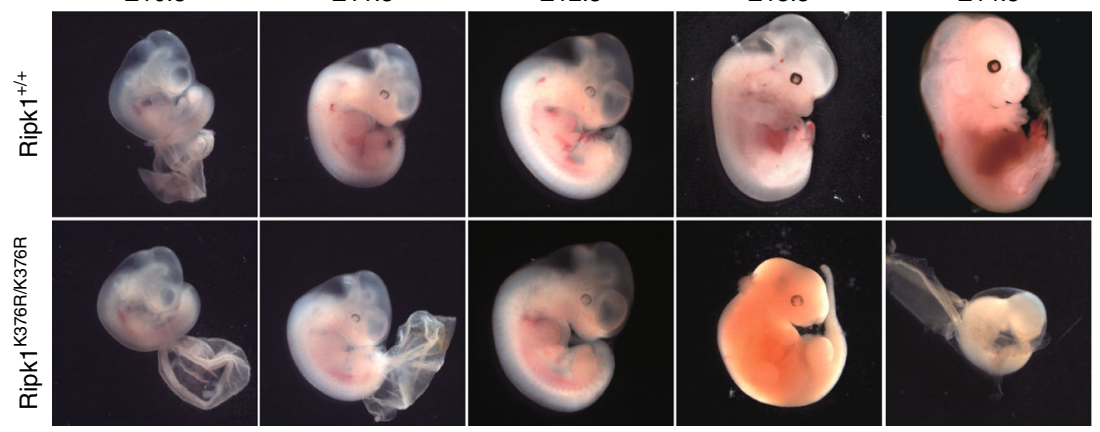

d
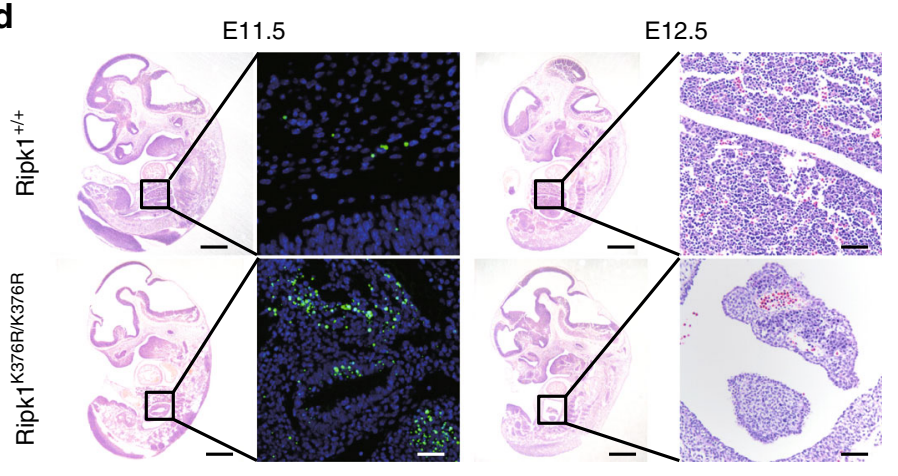

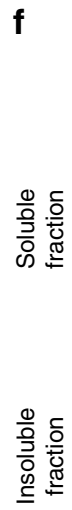

e

VE-cad $\mathrm{CC} 3$
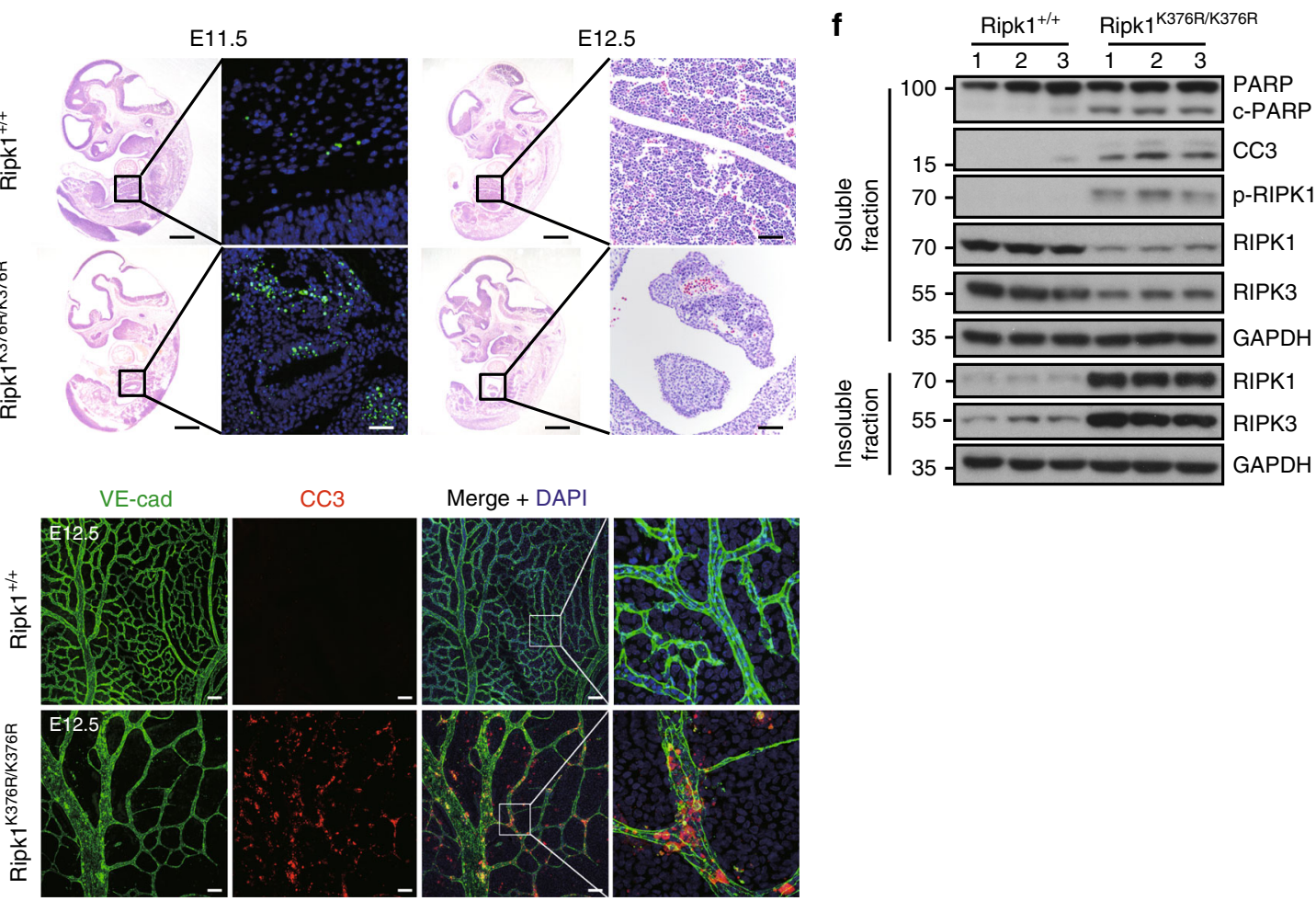

Fig. 1 Ripk $7^{K 376 R / K 376 R}$ mice die during embryogenesis. a Organization of the Ripk $7^{K 376 R}$ mutant allele. Lysine (AAG) was mutated to Arginine (AGA) at the 376 position in RIPK1. The mutation was confirmed by sequencing. $\mathbf{b}$ Observed numbers of embryos or live born pups of the indicated genotypes at various developmental stages from Ripk 7 K376R/+ mice intercrosses. Asterisk indicates the abnormal embryos. c Whole-mount dark field images of embryos with indicated genotypes. Images are representative of embryos from E10.5 $(n=5)$, E11.5 $(n=10)$, E12.5 $(n=16)$, E13.5 $(n=19)$, E14.5 ( $n=5)$. d Representative Hematoxylin and eosin (H\&E)-stained and TUNEL-stained fetal livers from mouse embryos of the indicated genotypes (Scale bars, $1 \mathrm{~mm}$ ). Images are representative of fetal livers from wild-type $(n=3)$ and Ripk $7376 R / K 376 R(n=3)$. Scale bars, $50 \mu \mathrm{m}$. e Immunostaining for VE-cad (green) and cleaved caspase-3(CC3) (red) on yolk sacs from wild-type and Ripk $7^{K 376 R / K 376 R}$ embryos. Scale bars, $100 \mu \mathrm{m}$. Images are representative of embryos from E12.5 $(n=3$ /genotype). $\mathbf{f}$ The body samples from E12.5 embryos were analyzed by western blot and immunoblotted for PARP, CC3, p-RIPK1(S166), RIPK1, and RIPK3 
embryos, indicating that the cell death induced by this mutation has effects on both embryonic tissues and yolk sacs (Fig. 1e). At E13.5 and E14.5, Ripk1 $1^{\text {K376R/K376R }}$ embryos were anemic with apparent developmental abnormalities which indicate the death of the mutant embryos (Fig. 1c). Therefore, these results suggest that germline mutation of $R i p k 1^{K 376 R / K 376 R}$ causes embryonic lethality at E12.5 with excessive cell death including apoptosis and necroptosis.

K376R blocks the ubiquitination of RIPK1. To investigate the contribution of RIPK1 K376 on diverse signaling, we stimulated primary MEFs from Ripk $1^{K 376 R / K 376 R}$ mice and Ripk $1^{+/+}$mice with TNF- $\alpha$. Within 5 min of TNF- $\alpha$ stimulation, obvious ubiquitination of RIPK1 in complex I was detected in Ripk $1^{+/+}$cells, whereas a significant reduction in RIPK1 ubiquitination was observed in Ripk1 $1^{K 376 R / K 376 R}$ cells (Fig. 2a). Accordingly, the interaction between RIPK1 and NEMO was markedly weakened in Ripk1 $1^{K 376 R / K 376 R}$ cells (Fig. 2c). Pre-treating cells with Smac mimetics, a cIAP antagonist, remarkably inhibited the ubiquitination of RIPK1 in response to TNF- $\alpha$, suggesting that ubiquitination of RIPK1 on K376 was mainly mediated by E3 ubiquitin ligases cIAP1/2 (Fig. 2b). Immunoprecipitation of ubiquitin chains showed that K63 ubiquitination of RIPK1 was more profoundly affected by K376R mutation (Supplementary Fig. 1a), which was consistent with previous reports that showed the polyubiquitin chains on RIPK1 were linked primarily through K63 of ubiquitin $^{3,40}$. To determine if the ubiquitination of RIPK1 on K376 was required for NF- $\kappa B$ activation, we performed a timecourse experiment by treating cells with TNF- $\alpha$. In Ripk $1^{+/+}$cells, I $\kappa \mathrm{B} \alpha$ was phosphorylated within $5 \mathrm{~min}$ in response to TNF- $\alpha$ and was rapidly degraded (Fig. 2d). Consequently, phosphorylation and gradual translocation of p65 were observed (Fig. 2d, e), which activated the transcription of target genes (Fig. 2f). In contrast, in Ripk $1^{K 376 R / K 376 R}$ cells, IkB $\alpha$ activation was dramatically blocked, and subsequent translocation of p65 was delayed which led to impaired transcriptional induction of NF- $\kappa \mathrm{B}$-targeted genes (Fig. $2 \mathrm{~d}-\mathrm{f})$. Importantly, except for $\mathrm{NF}-\kappa \mathrm{B}$ pathway, other signaling pathways in Ripk $1^{K 376 R / K 376 R}$ cells such as activation of JNK, p38, and Erk1/2 were almost unaffected (Fig. 2d), indicating a distinctive role of $\mathrm{RIPK} 1^{\mathrm{K} 376 \mathrm{R}}$ in activating NF- $\mathrm{BB}$ signaling in MEFs. Thus, our results show a critical role of $\mathrm{K} 376$ in mediating RIPK1 ubiquitination and NF- $\kappa B$ activation in response to TNF- $\alpha$ stimulation.

RIPK1 ${ }^{\text {K376R }}$ promotes both apoptosis and necroptosis. Defective polyubiquitination of RIPK1 has been proposed to promote TNF- $\alpha$ induced apoptosis in Jurkat $\mathrm{T}$ cells $\mathrm{s}^{3,5,38}$. However, in MEFs, we found that the disruption of RIPK1 ubiquitination mediated by K376R sensitized cells to not only apoptosis but also necroptosis. In response to TNF- $\alpha$, much more Ripk $1^{K 376 R / K 376 R}$ cells underwent cell death compared to Ripk $1^{+/+}$control cells (Fig. 3a). After $24 \mathrm{~h}$ of TNF- $\alpha$ treatment, cleaved caspase- 3 and phosphorylated MLKL were clearly detected in Ripk $1^{K 376 R / K 376 R}$ cells, indicating the simultaneous occurrence of apoptosis and necroptosis (Fig. 3b). In line with these results, mutant RIPK1 $1^{\mathrm{K} 376 \mathrm{R}}$ was found in complex II after TNF- $\alpha$ stimulation for $12 \mathrm{~h}$, suggesting that RIPK1 $1^{\mathrm{K} 376 \mathrm{R}}$ promoted RIPK1 transition from complex I to complex II which led to the subsequent apoptosis and necroptosis (Fig. 3d). Besides, stimulating MEFs with TNF-a combined with Smac mimetics (TS), Ripk1 K376R/K376R cells showed more sensitivity than the Ripk $1^{+/+}$cells did (Fig. 3a). In the presence of caspase inhibitor zVAD-fmk (Z), TS induced higher level of necroptosis in $R i p k 1^{K 376 R / K 376 R}$ MEFs compared with $R i p k 1^{+/+}$cells as the oligomerization of phosphorylated
MLKL and aggregation of RIPK1 and RIPK3 were detected in Ripk $1^{K 376 R / K 376 R}$ MEFs earlier than in Ripk1 ${ }^{+/+}$MEFs (Fig. 3a, c).

We next investigated the effect of NF- $\kappa B$ signaling defects in $R i p k 1^{K 376 R / K 376 R}$ MEFs on its sensitivity to cell death. Interestingly, activating $\mathrm{NF}-\kappa \mathrm{B}$ signaling by $\mathrm{P} 65^{\mathrm{S} 275 \mathrm{D}}$ overexpression failed to rescue mutant cells from TNF- $\alpha$ induced apoptosis and necroptosis (Supplementary Fig. 1b). Moreover, when treated with TNF- $\alpha$ combined with cycloheximide (CHX) which blocked NF$\kappa \mathrm{B}$-dependent gene expression, Ripk1 ${ }^{K 376 R / K 376 R} \mathrm{MEFs}$ were still more sensitive to cell death than the Ripk $1^{+/+}$control (Fig. 3a). These results argued against NF- $\kappa B$ defects being the leading cause for enhanced cell death in mutant cells. Therefore, we conclude that $\mathrm{RIPK} 1^{\mathrm{K} 376 \mathrm{R}}$ promotes the formation of complex II to facilitate the induction of apoptosis and necroptosis.

RIPK1 ${ }^{\mathrm{K} 376 \mathrm{R}}$ induced cell death relies on its kinase activity. It has been shown that the activation of RIPK1 kinase activity, which promotes autophosphorylation of RIPK1, is critical for the transition from complex I to complex $\mathrm{II}^{29}$. Using phosphoSer166-RIPK1 (p-RIPK1(S166)) antibody as a marker of RIPK1 activation, we found more phosphorylated RIPK1 in both complex I and complex II in TNF- $\alpha$-stimulated Ripk1 $1^{K 376 R / K 376 R}$ MEFs (Figs. 2a, 3d). Thus, we wondered whether RIPK1 ${ }^{\mathrm{K} 376 \mathrm{R}}$ -induced cell death relied on the kinase activity of RIPK1. Treatment of Necrostatin-1(Nec-1s or N), a RIPK1 kinase inhibitor, efficiently prolonged the survival of Ripk $1^{K 376 R / K 376 R} \mathrm{MEFs}$ in response to TNF-a (or TC or TS) induced apoptosis and necroptosis (Fig. 4a). Similar results were obtained from treatments of TSZ or TCZ (Fig. 4a). Consistently, we found that the level of p-Ser166 RIPK1 was elevated in mutant cells and was significantly blocked by Nec-1s (Fig. 5c). Consequently, levels of cleaved caspase- 3 and p-MLKL were reduced as well, indicating that RIPK1 ubiquitination on K376 is required for suppressing RIPK1 kinase-mediated the apoptosis and necroptosis (Fig. $5 \mathrm{c}$ ).

As we observed higher levels of p-RIPK1 in Ripk1 $1^{K 376 R / K 376 R}$ embryos (Fig. 1e), we next investigated the effect of Nec-1s on the $R i p k 1^{K 376 R / K 376 R}$ lethality in vivo. Feeding heterozygous mothers with Nec-1s during gestation period effectively prevented the lethal phenotypes of homozygous descendants (Fig. 4b). Compared with Ripk1 $1^{K 376 R / K 376 R}$ embryos without Nec-1s treatment which were dead and anemic at E13.5, Nec-1s-treated mutant embryos appeared much more normal although their yolk sacs were not completely rescued (Fig. 4b). Histological examination showed that Nec-1s treatment profoundly prevented the tissue losses in Ripk1 $1^{K 376 R / K 376 R}$ embryos (Fig. 4c). Besides, immunoblot analysis confirmed that RIPK1 phosphorylation and apoptosis featured by CC3 in mutant embryos were inhibited by Nec-1s treatment (Fig. 4f). Consistently, the number of TUNEL positive cells in the fetal livers of $R i p k 1^{K 376 R / K 376 R}$ embryos was significantly reduced by Nec-1s treatment, suggesting that the massive cell death was largely restrained by RIPK1 kinase inhibition (Fig. 4d, e). Taken together, these results suggest

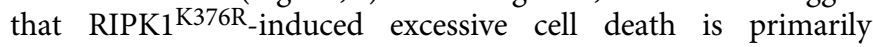
dependent on the kinase activity of RIPK1.

Ripk1 ${ }^{K 376 R /-}$ mice are viable but develop severe inflammation. Assuming that RIPK1 activation was responsible for the cell death in $R i p k 1^{K 376 R / K 376 R}$ embryos, we next tested whether the amount of activated RIPK1 had an impact on this lethality. By crossing Ripk $1^{K 376 R /+}$ into Ripk $1^{+/-}$mice, we generated Ripk $1^{K 376 R /-}$ mice with only half amounts of RIPK 1 K376R . Strikingly, in contrast to $R i p k 1^{K 376 R / K 376 R}$ mice which were totally abnormal at E13.5, Ripk $1^{K 376 R /-}$ mice developed normally (Figs. 1c, 5a). MEFs isolated from Ripk1 $1^{K 376 R /-}$ embryos were less sensitive to TNF- $\alpha$ induced apoptosis and necroptosis compared to Ripk $1^{\text {K376R/K376R }}$ 
a

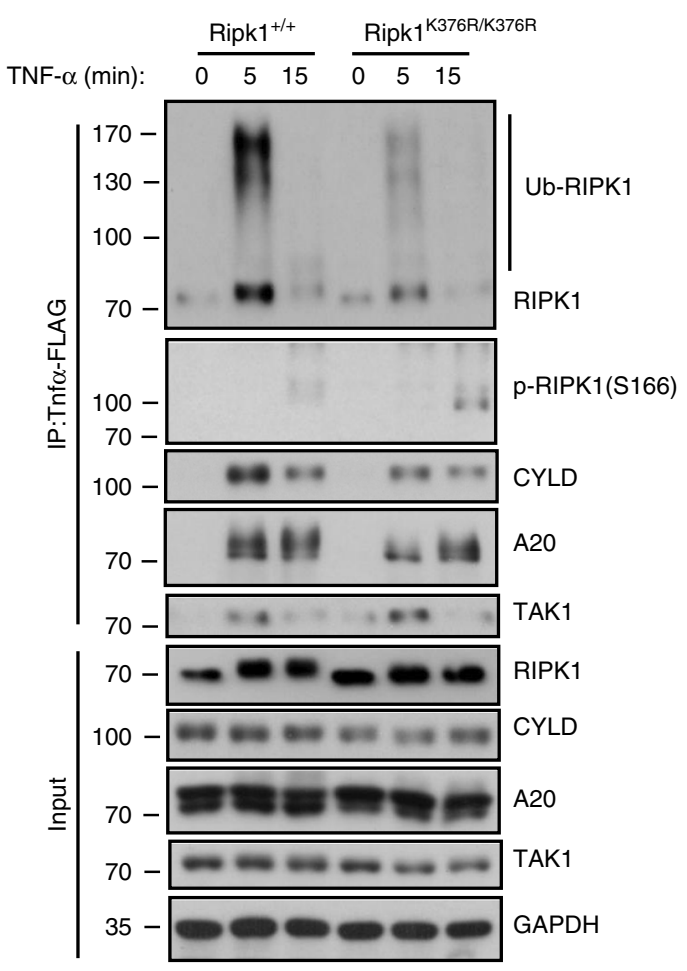

d

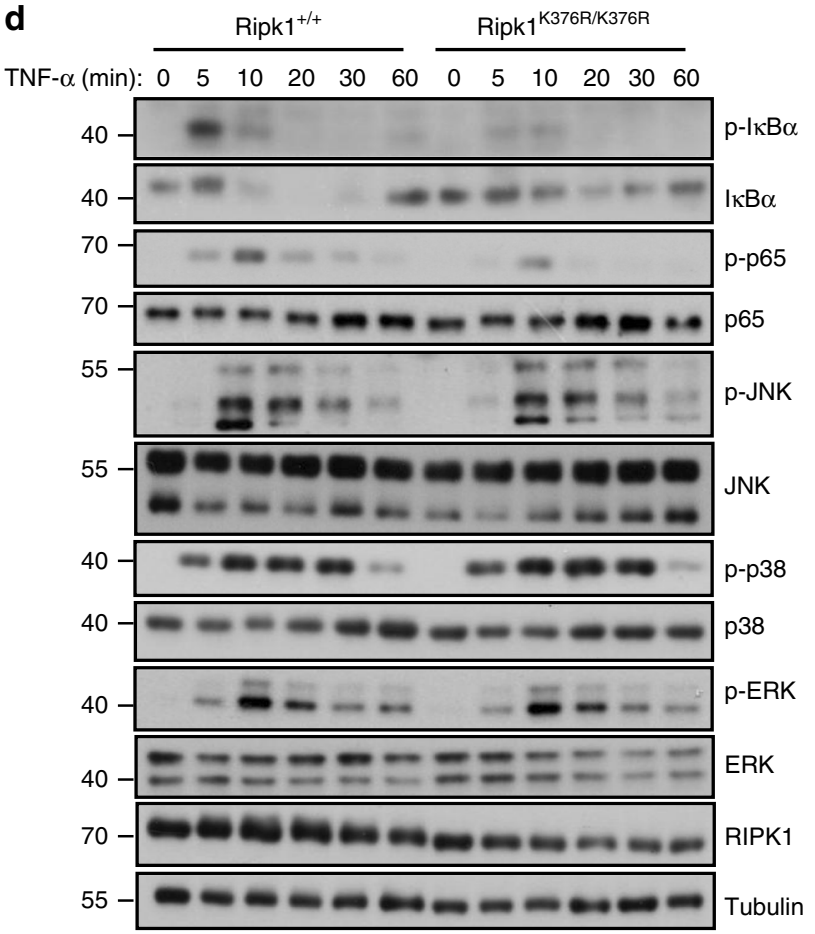

b

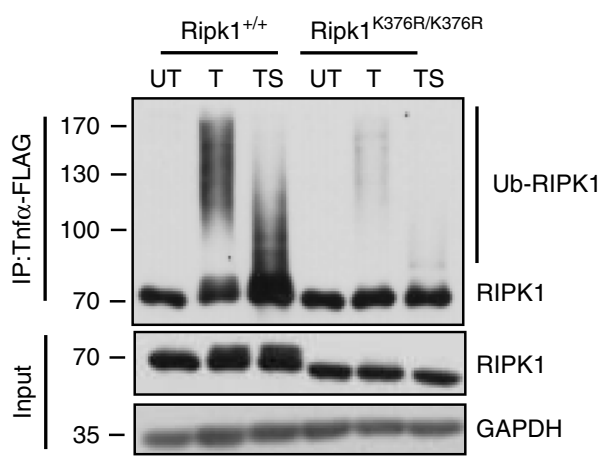

C

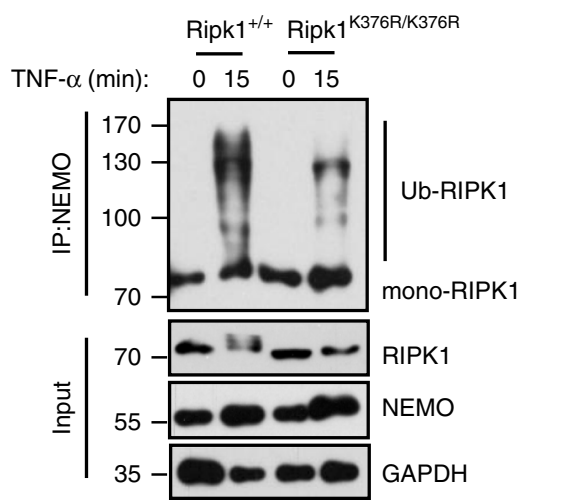

e

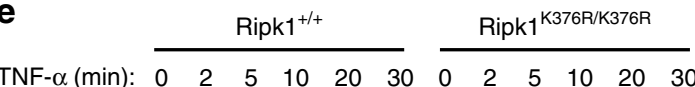

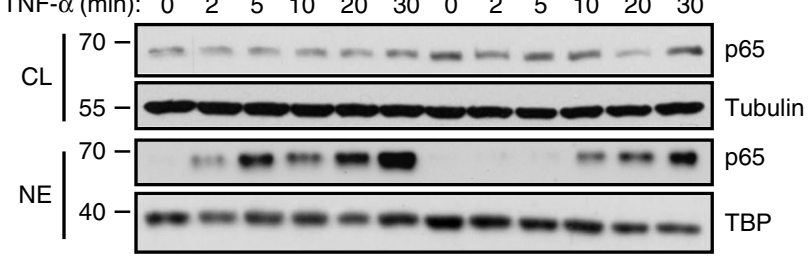

f

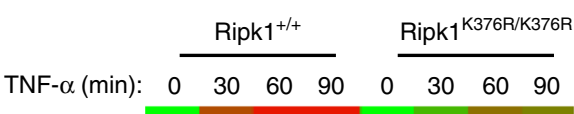

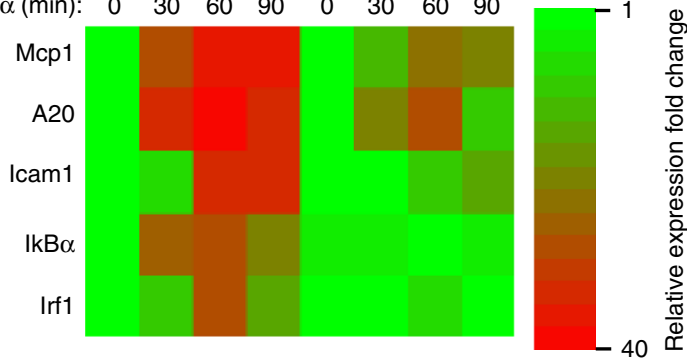

Fig. 2 K376R blocks the ubiquitination of RIPK1 and NF-KB activation upon TNF- $\alpha$ stimulation. a Ripk $7^{+} /+$and Ripk $7^{K 376 R / K 376 R}$ MEFs were treated with FlagTNF- $\alpha(100 \mathrm{ng} / \mathrm{ml})$ for the indicated periods of time, complex I was immnoprecipitated using anti-FLAG beads, endogenous RIPK1 ubiquitination and indicated proteins were detected by western blotting. b Ripk1+/+ and Ripk $1^{K 376 R / K 376 R}$ MEFs were stimulated by Flag-TNF- $\alpha$ (100 ng/ml) for $5 \mathrm{~min}$ with or without pretreatment of Smac mimetics, complex I was immunoprecipitated using anti-FLAG beads, endogenous RIPK1 ubiquitination were detected by western blotting. Abbreviations are as follows: T Flag-TNF- $\alpha$ stimulation, TS Flag-TNF- $\alpha$ stimulation with Smac mimetics pretreatment. c Ripk $1^{+/}+{ }_{\text {and }}$ Ripk $7^{K 376 R / K 376 R}$ MEFs were treated with TNF- $\alpha(100 \mathrm{ng} / \mathrm{ml})$ for $15 \mathrm{~min}$ and then NEMO was immunoprecipitated. Ubiquitinated RIPK1 was detected with RIP1 antibody. Cell lysates were analyzed by western blotting using the indicated antibodies. d Ripk $1^{+} /+$and Ripk ${ }^{K 376 R / K 376 R}$ MEFs were treated with TNF- $\alpha$ $(20 \mathrm{ng} / \mathrm{ml})$ for the indicated periods of time, signaling pathways including NF- $\mathrm{\kappa B}, \mathrm{JNKs}, \mathrm{p} 38$, and ERKs were examined by western blotting. e Ripk ${ }^{+} /+$and Ripk ${ }^{K 376 R / K 376 R}$ MEFs were treated with TNF- $\alpha(20 \mathrm{ng} / \mathrm{ml})$ for $5 \mathrm{~min}$. P65 nuclear translocation was examined by western blotting. Abbreviations are as follows: NE nuclear extracts, CL cell lysates. f Ripk1+/+ and Ripk7K376R/K376R MEFs were treated with TNF- $\alpha$ (20 ng/ml) for the indicated times, mRNA transcription change of NF- $\mathrm{KB}$ targeted genes were determined by quantitative PCR ( $\mathrm{qPCR}$ ) 
a
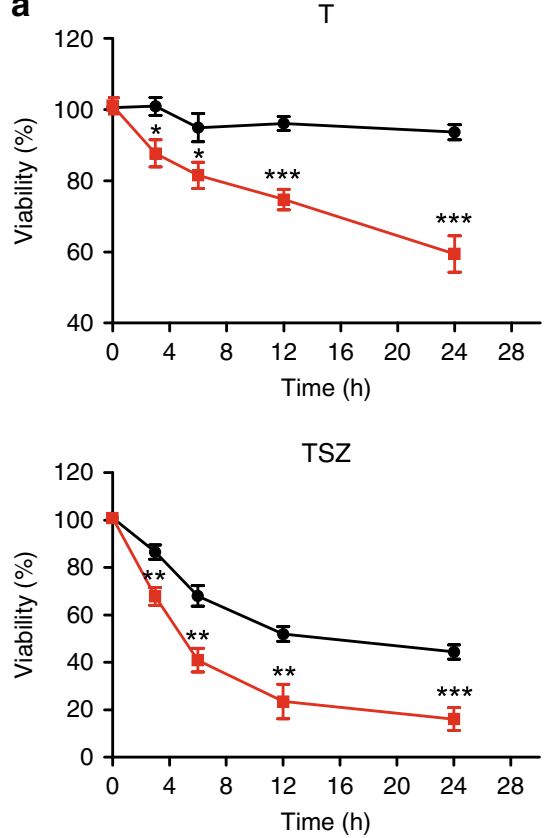

b
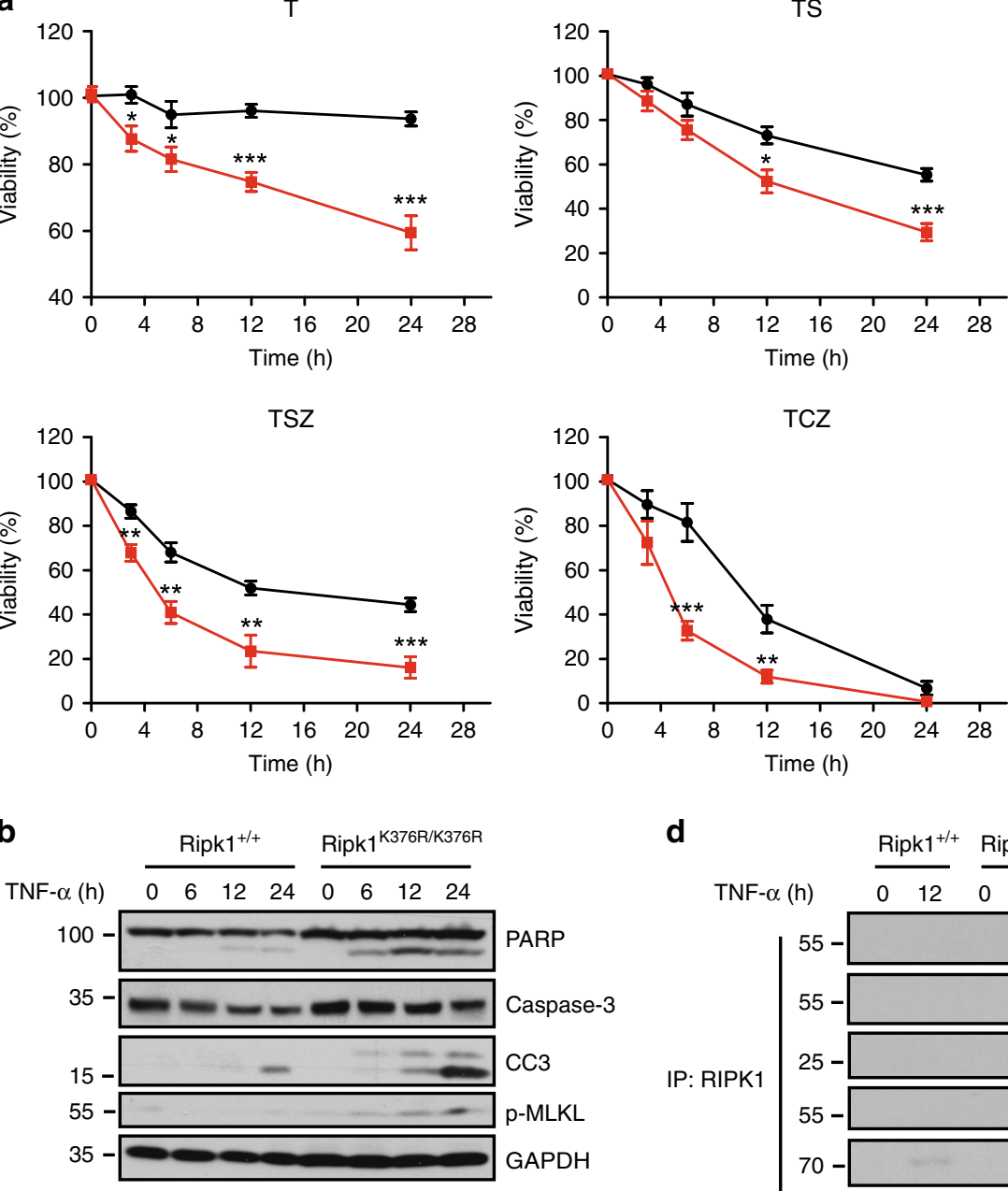

d

PARP

Caspase-3

CC3

p-MLKL

GAPDH

IP: RIPK1

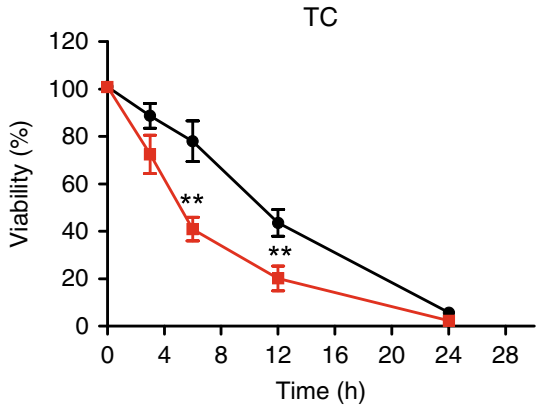

$\rightarrow \operatorname{Ripk}^{+/+}$

C
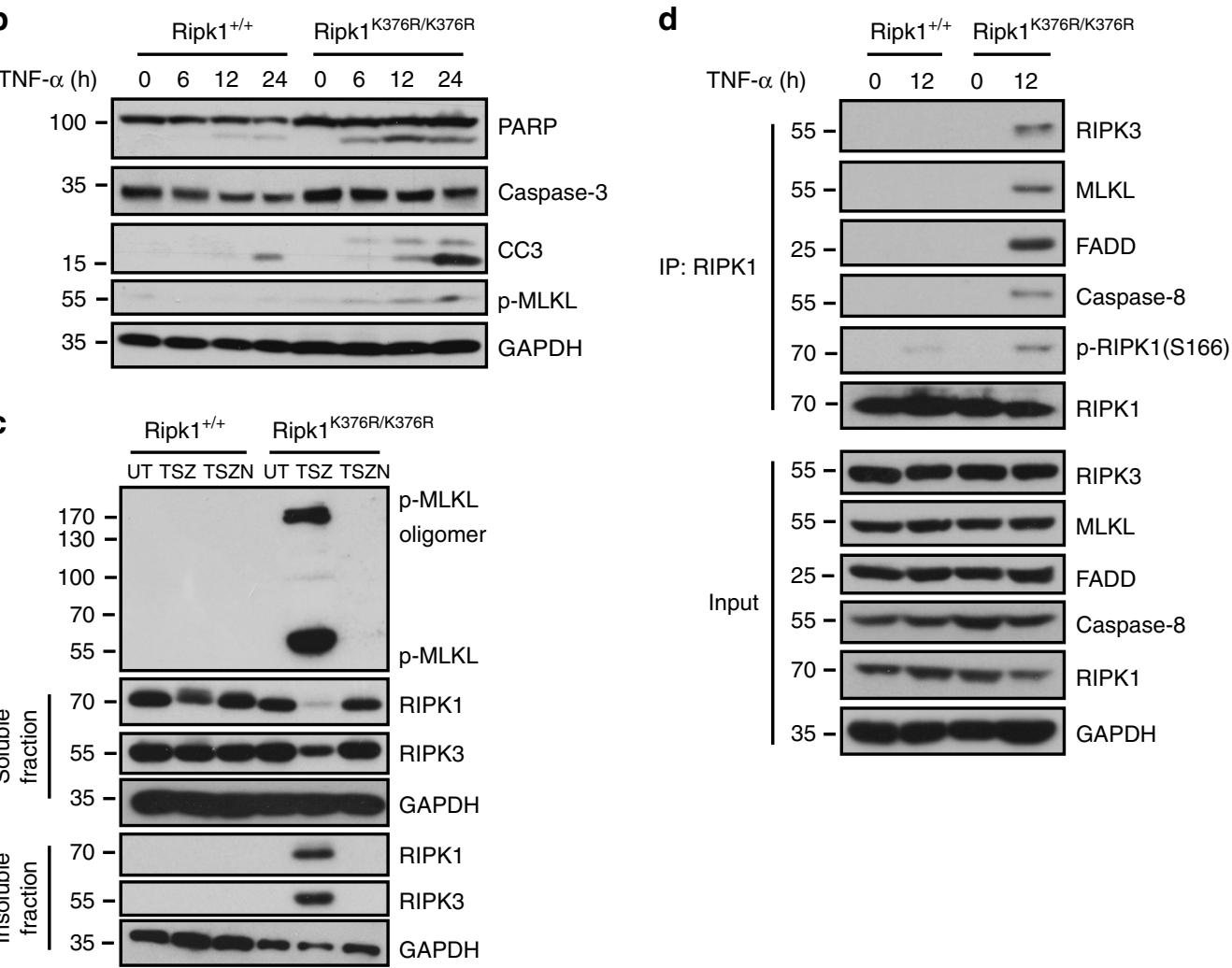

Fig. 3 Cells bearing RIPK1K376R/K376R are more susceptible to TNF- $\alpha$ induced apoptosis and necroptosis. a Ripk1+/+ and Ripk1K376R/K376R MEFs were treated with T, TS, TSZ, TC, TCZ for indicated times, respectively. Cell viability was determined using the CellTiter-Glo kit. The data are represented as the mean \pm SEM of MEFs derived from 3 embryos. $P$ values were determined by Student's $t$-test $\left({ }^{\star} p<0.05,{ }^{\star \star} p<0.01,{ }^{\star \star \star} p<0.001\right)$. Abbreviations are as follows: UT untreated, TSZ TNF- $\alpha(20 \mathrm{ng} / \mathrm{ml})+$ Smac mimetic $(100 \mathrm{nM})+z \mathrm{VAD}(20 \mu \mathrm{M})$; TSZN, TNF- $\alpha+$ Smac mimetic $+z V A D+N e c r o s t a t i n-1(20 \mu M)$; TC, TNF- $\alpha+\mathrm{CHX}(20 \mathrm{ng} / \mathrm{ml}) ; \mathrm{TCZ}, \mathrm{TNF}-\alpha+\mathrm{CHX}+\mathrm{zVAD}$. Source data are provided as a Source Data file. b Ripk1+/+ and Ripk7K376R/K376R MEFs were treated with mouse TNF- $\alpha(20 \mathrm{ng} / \mathrm{ml})$ for indicated times, the cell lysates were analyzed by western blotting using the indicated antibodies. $\mathbf{c}$ Ripk $7^{+/}+{ }$and Ripk $7^{K 376 R / K 376 R}$ MEFs were treated with TSZ or TSZN for $3 \mathrm{~h}$, the cell lysates were resolved on non-reducing gel and immunoblotted with anti-p-MLKL antibody. d Ripk $1^{+/+}$and Ripk $7^{K 376 R / K 376 R}$ MEFs were treated with mouse TNF- $\alpha$ ( $20 \mathrm{ng} / \mathrm{ml}$ ) for $12 \mathrm{~h}$, anti-RIPK1 was used to immunoprecipitate complex and immunocomplexes were analyzed by western blotting using indicated antibody 

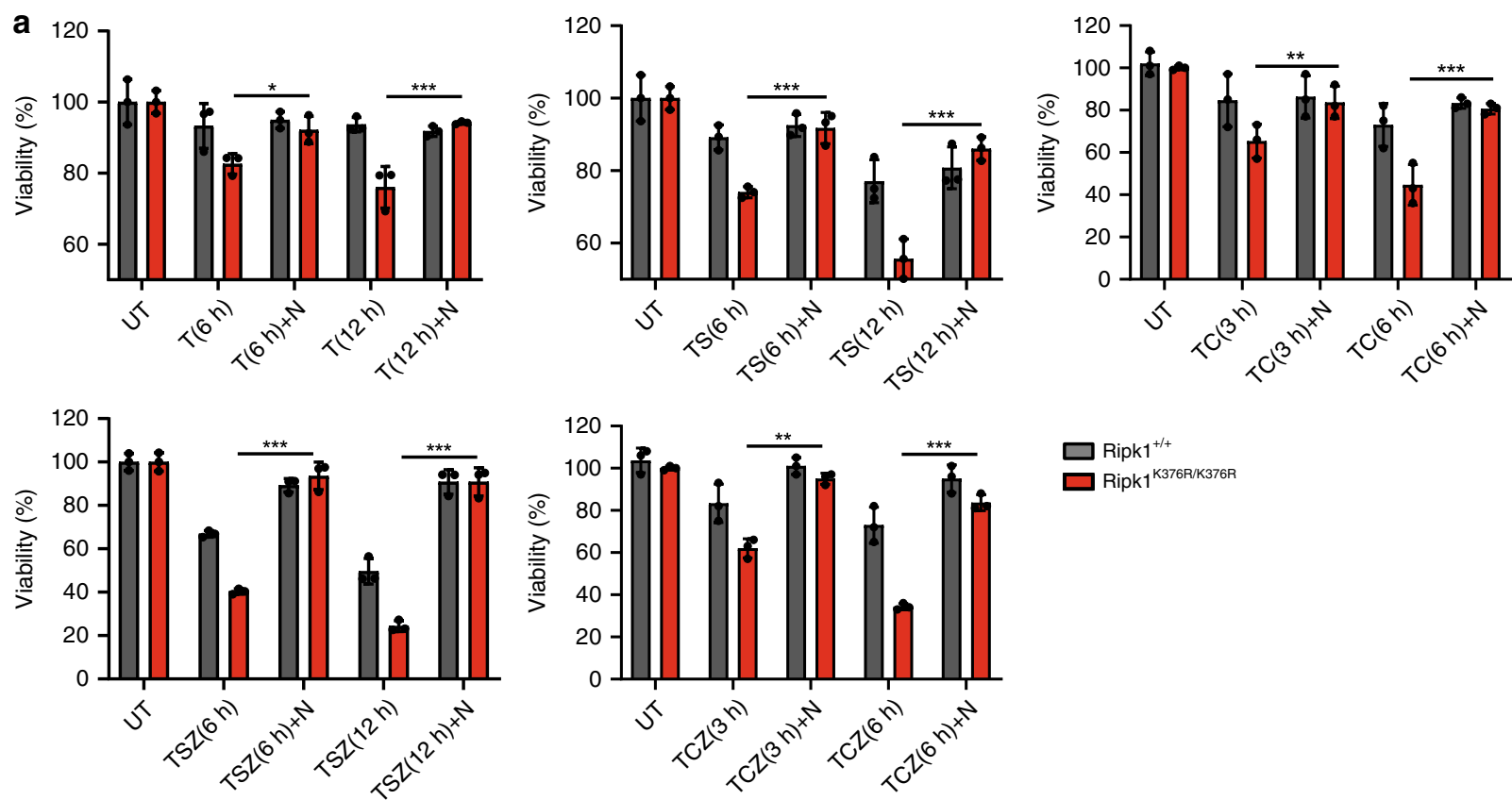

b
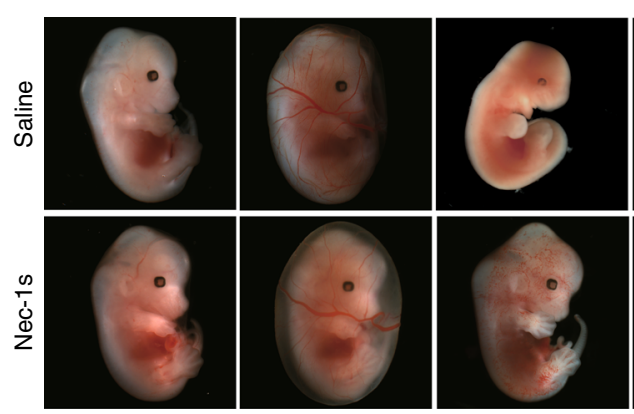

Ripk1 K376R/K376R
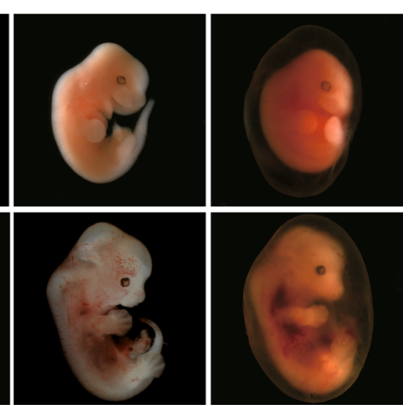

d

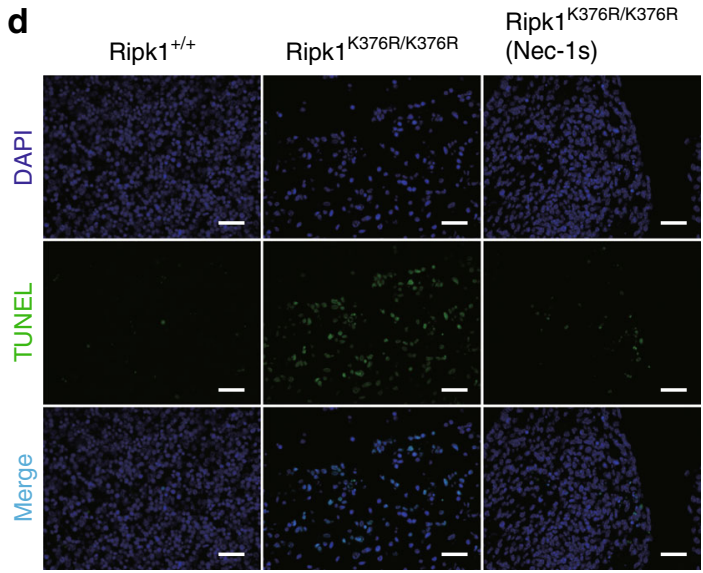

e

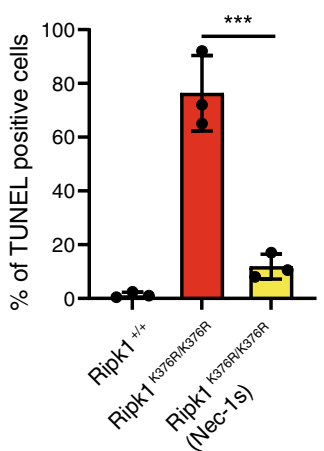

C

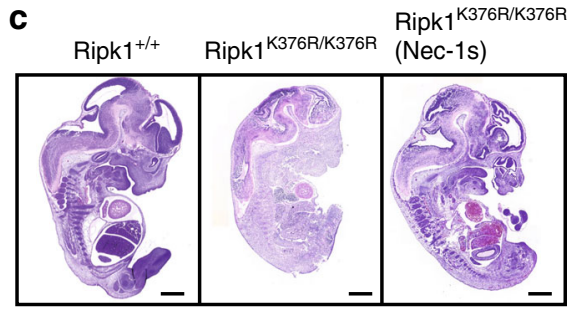

f

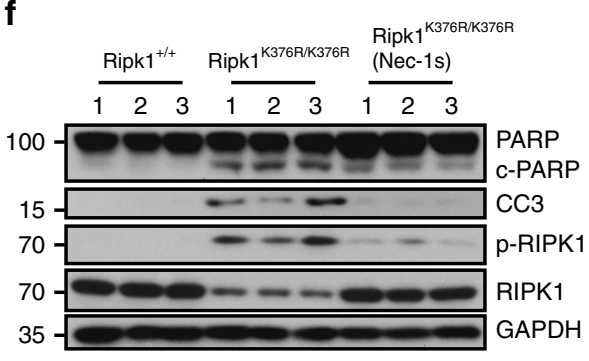

Fig. 4 Treatment of Nec-1s inhibits cell death induced by RIPK1 K376R. a Ripk1+/+ and Ripk $7^{K 376 R / K 376 R}$ MEFs were treated with T, TS, TSZ, TC, TCZ for indicated times with or without Nec-1s as indicated. Cell viability was determined using the CellTiter-Glo kit. The data are represented as the mean \pm SEM $(n=3)$. $P$ values were determined by Student's t-test $\left({ }^{\star} p<0.05,{ }^{* \star} p<0.01,{ }^{\star \star \star} p<0.001\right)$. Source data are provided as a Source Data file. $\mathbf{b}$ Pregnant Ripk $7^{K 376 R /+}$ females were fed with Nec-1s $(50 \mathrm{mg} / \mathrm{kg}$ ) or saline solution once per day since day 9.5 of gestation and sacrificed on day 13.5. Representative embryos with indicated genotypes were presented. c Representative H\&E staining (scale bars, $1 \mathrm{~mm}$ ) and d TUNEL staining of fetal livers from mouse embryos of the indicated genotypes. Each image is representative of at least three embryos. Scale bars, $50 \mu \mathrm{m}$. e Quantification of TUNEL positive cells in Fig. 4 d. The results are represented as \pm SEM of three embryos per group. $P$ value was calculated by Student's $t$-test $\left({ }^{\star \star \star} p<0.001\right)$. Source data are provided as a Source Data file. f The fetal liver samples from three embryos per group were analyzed by western blot and immunoblotted with PARP, CC3, p-RIPK1(S166), and RIPK1 
a

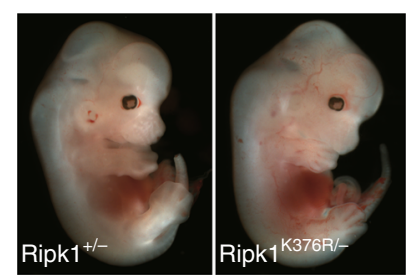

C

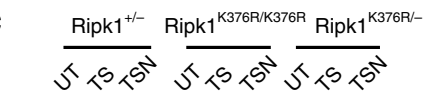

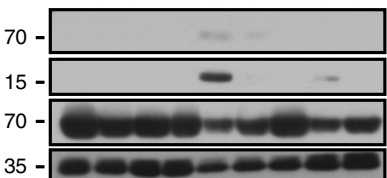

p-RIPK1(S166) CC3

\section{RIPK1} GAPDH

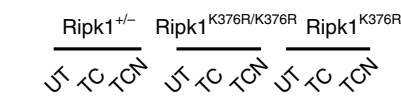

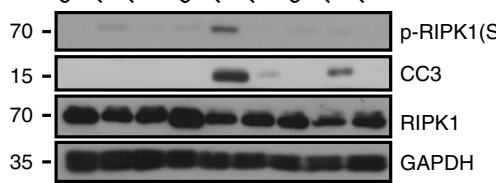

d

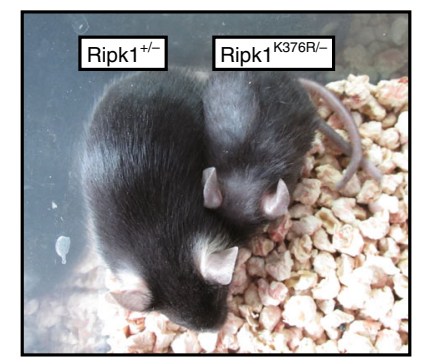

f

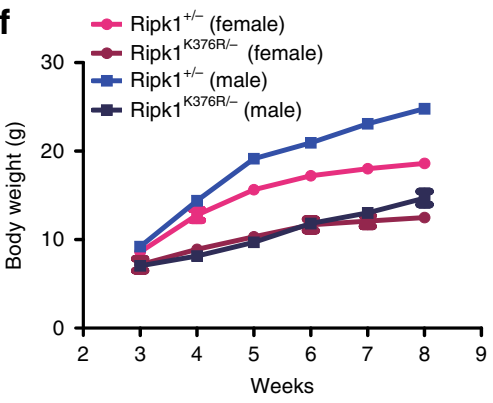

b 120

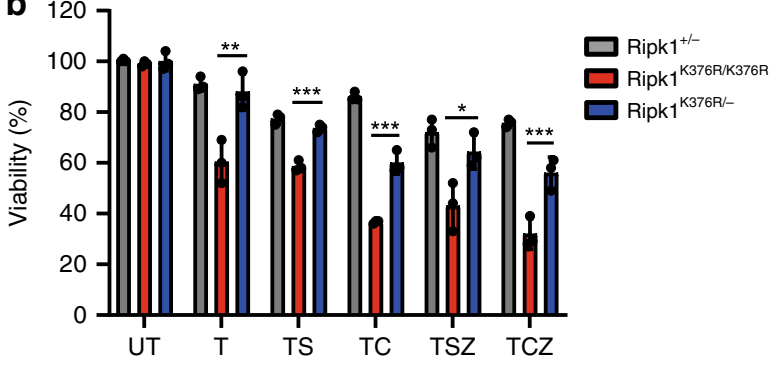

Ripk1 ${ }^{+/-}$Ripk1 ${ }^{\text {K376/K376R }}$ Ripk1 $1^{\text {K376R/- }}$

$$
35 \text { - } \mathrm{GAPDH}
$$

$\mathrm{Ripk}^{\text {+/- }}$ Ripk1 $^{\text {K376R/K376R }}$ Ripk1 $^{\text {K376R/- }}$

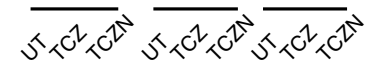

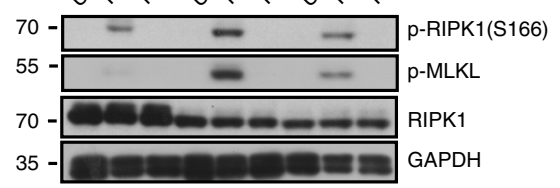

e
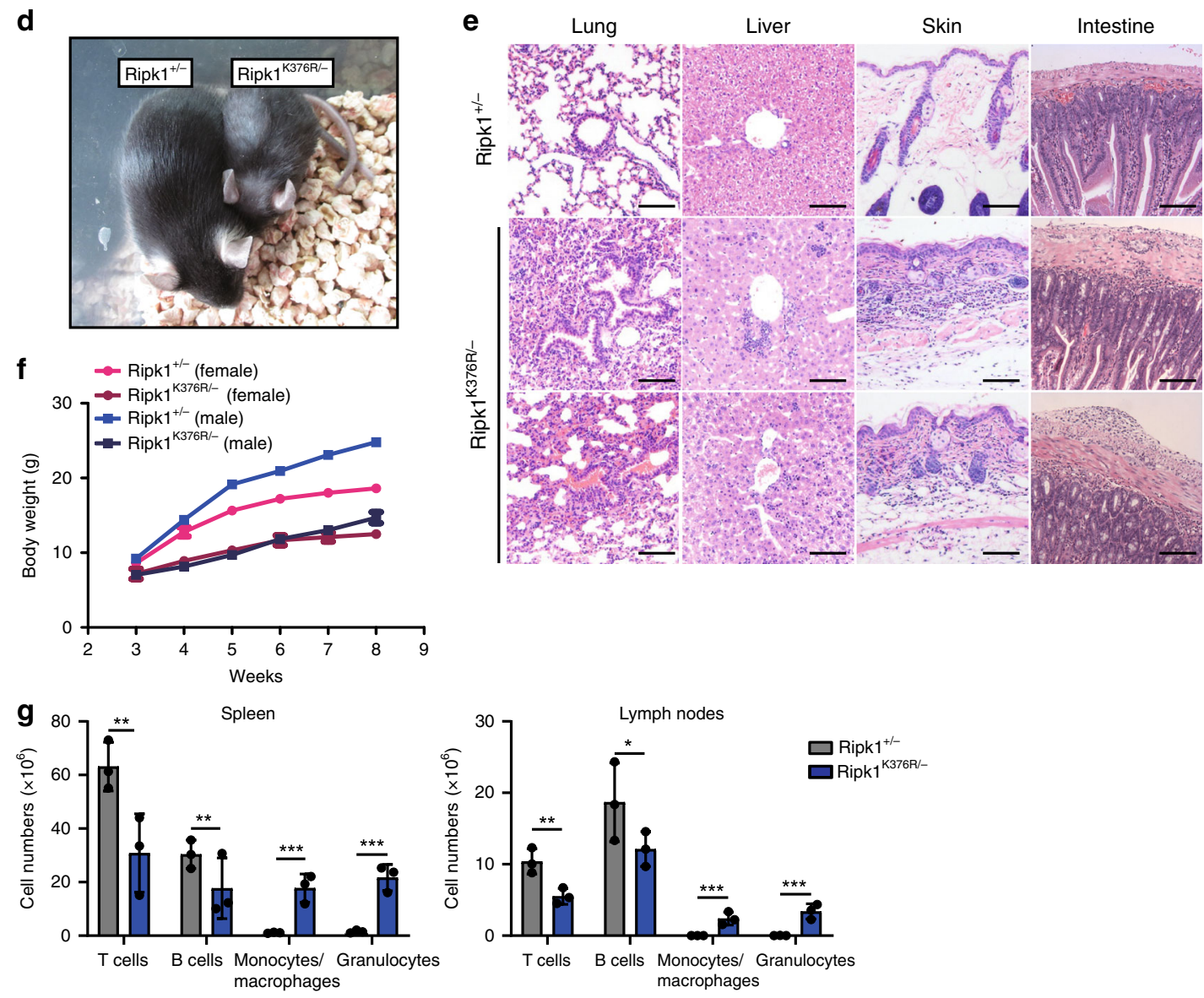

MEFs (Fig. 5b). Similar results were obtained when treating cells with TC, TS, TCZ, and TSZ (Fig. 5b). In line with these data, the high levels of p-RIPK1(S166) with cleaved caspase- 3 and phosphorylation of MLKL detected in Ripk1 $1^{K 376 R / K 376 R}$ MEFs were profoundly reduced in Ripk1 $1^{K 376 R /-}$ MEFs (Fig. 5c). These results suggest that there is likely to be a threshold above which activated RIPK $1{ }^{\mathrm{K} 376 \mathrm{R}}$ can trigger the lethal pathways during embryogenesis. Intriguingly, Ripk $1^{K 376 R /-}$ mice can grow up into adulthood, although they displayed much smaller and had lower body weight than their littermate controls (Fig. 5d, f). Histological 
Fig. 5 Ripk 1 K376R/- mice are viable but display systemic inflammation. a Representative whole-mount dark field images of E13.5 embryos with indicated

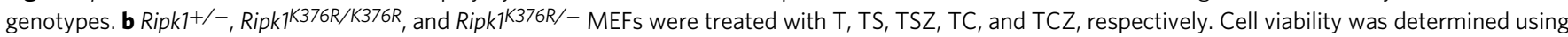
the CellTiter-Glo kit. Data are represented as mean \pm SEM of MEFs derived from three embryos. $P$ values were determined by Student's $t$-test $\left({ }^{*} p<0.05\right.$, $\left.{ }^{\star \star} p<0.01,{ }^{\star \star \star} p<0.001\right)$. Abbreviations are as follows: UT untreated, TSZ TNF- $\alpha(20 \mathrm{ng} / \mathrm{ml})+$ Smac mimetic $(100 \mathrm{nM})+z V A D(20 \mu M), T S Z N$ TNF- $\alpha$ + Smac mimetic $+z V A D+N e c r o s t a t i n-1(20 \mu M), T C$ TNF- $\alpha+C H X(20 \mathrm{ng} / \mathrm{ml})$, TCZ TNF- $\alpha+C H X+z V A D$. Source data are provided as a Source Data file. c Ripk1+/-, Ripk7 T376R/K376R, and Ripk7 T376R/- MEFs were treated with TS(6h), TSZ(2h), TC(3h), TCZ(1h) with or without Nec-1s. Western blottings of indicated proteins were shown. d Representative 8-week-old Ripk 7 K376R/K376R mouse and littermate control. e Representative hematoxylin and eosin (H\&E)-stained tissues from 4-week-old animals of the indicated genotypes. (Scale bars, $100 \mu \mathrm{m}$ ). f Plot of weight of littermate Ripk $1^{+/-}$and Ripk $7^{K 376 R /-}$ mice. $\mathbf{g}$ Different cell subsets in spleens and LNs from 6-week-old mice with indicated genotypes were determined by flow cytometry using following markers: B cells $\left(\mathrm{CD} 19^{+}\right.$or $\left.\mathrm{B} 220^{+}\right)$, T cells $\left(\mathrm{CD}^{+}\right)$, Monocytes and macrophages (CD11b $\left.{ }^{+}\right)$, Granulocytes $\left(\mathrm{Gr}-1^{+}\right)$. Data are represented as mean $\pm \mathrm{SEM}$ $\left(n=3\right.$ /genotype). $P$ values were calculated by Student's $t$-test $\left({ }^{\star} p<0.05,{ }^{\star \star} p<0.01,{ }^{\star \star \star} p<0.001\right)$. Source data are provided as a Source Data file

examination of 4 -week-old to 8 -week-old Ripk1 $1^{K 376 R /-}$ mice revealed signs of inflammation in multiple organs including skins, lungs, intestines, and livers (Fig. 5e). Flow cytometric analysis of Ripk $1^{K 376 R /-}$ spleens and lymph nodes revealed decreased numbers of $\mathrm{T}$ cells $\left(\mathrm{CD}^{+}\right)$and B cells $\left(\mathrm{B} 220^{+}\right.$or $\left.\mathrm{CD} 19^{+}\right)$while remarkably increased numbers of granulocytes $\left(\mathrm{Gr}-1^{+}\right)$and macrophages $\left(\mathrm{CD}_{11 \mathrm{~b}}{ }^{+}\right)$(Fig. 5g), indicating that Ripk $1^{\mathrm{K} 376 R /-}$ mice developed systemic inflammation after birth. Collectively, these data suggest that K376-mediated RIPK1 ubiquitination is critical for restraining inflammation during postnatal development.

Loss of $T n f r 1$ rescues the lethality of $R i p k 1^{K 376 R / K 376 R}$ mice. To further investigate the upstream signaling inducing the Ripk1 $1^{K 376 R / K 376 R}$ lethality, we crossed Ripk1 $1^{K 376 R}$ into Tnfr $1^{-/-}$ or Ifnar ${ }^{-/-}$background to generate Tnfr1 ${ }^{-/-}$Ripk $1^{\text {K376R/K376R }}$ and Ifnar ${ }^{-/}$Ripk $1^{K 376 R / K 376 R}$ mice, respectively. Results from timed pregnancies showed that deletion of $T n f r 1$ fully rescued the Ripk $1^{K 376 R / K 376 R}$ lethality during embryonic development (Fig. $6 \mathrm{a}-\mathrm{c})$. In contrast, ablation of Ifnar had no effect on the Ripk1 $1^{K 376 R / K 376 R}$ embryos and failed to rescue them from embryonic lethality (Fig. 6a). Consistent with these observations, the numbers of TUNEL-positive cells were remarkably increased and cleaved caspase- 3 was clearly detected in the fetal livers of Ifnar $^{-/}$Ripk $^{K 376 R / K 376 R}$ embryos (Supplementary Fig. 2a, b). Thus, these results suggest that TNFR1, rather than IFNR1, induces signaling that triggers the death of Ripk1 ${ }^{K 376 R / K 376 R}$ mice during embryogenesis.

Although ablation of Tnfrl prevented the embryonic death of

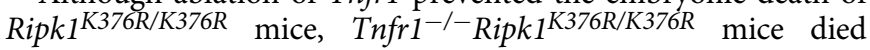
around 2 weeks after birth (Fig. 6h). These mice succumb to systemic inflammation as obvious inflammatory cell infiltrations were observed in the skin, lung, liver, and intestine (Fig. 6e). Quantitative RT-PCR examination revealed enhanced levels of inflammatory cytokines in the lungs and intestines of $\mathrm{Tnfr} 1^{-/-}$ Ripk $1^{K 376 R / K 376 R}$ mice (Fig. 6d). In addition, the composition of immune cells in spleen and thymus of mutant mice was largely disrupted. Compared with $\operatorname{Tnfr} 1^{-/-}$controls, the numbers of lymphocytes $\left(\mathrm{CD}^{+}\right.$cells and $\mathrm{B}^{+} 20^{+}$cells) in the spleen were reduced, whereas the numbers of myeloid cells $\left(\mathrm{CD} 11 \mathrm{~b}^{+}\right.$or $\mathrm{Gr}-1^{+}$ cells) were greatly increased (Supplementary Fig. 2d, e). In the thymus, the total cell counts were dramatically reduced with abnormal compartments of $\mathrm{T}$ cells in which $\mathrm{CD} 4^{+}$and $\mathrm{CD} 8^{+}$ single-positive populations were augmented while $\mathrm{CD} 4^{+} \mathrm{CD} 8^{+}$ double-positive population was decreased (Supplementary Fig. $2 \mathrm{~d}, \mathrm{e})$. These results showed that the homeostasis of the immune system in $T n f r 1^{-/-}$Ripk $1^{K 376 R / K 376 R}$ mice was disrupted, suggesting that K376-mediated ubiquitination of RIPK1 also contributes to regulating immune homeostasis.

We then sought to address the cause for the postnatal death of Tnfr $1^{-1-}$ Ripk1 K376R/K376R. Histological examination showed multiple tissues from $\operatorname{Tnfr} 1^{-/-}$Ripk1 K376R/K376R mice displayed tissue loss and abnormal architecture, indicating that these phenotypes may be a result of massive cell death (Fig. 6e). Using cleaved caspase- 3 as a marker for apoptosis, we observed no significantly positive cells detected in skins, livers and intestines of $\operatorname{Tnfr} 1^{-/}$Ripk $1^{K 376 R / K 376 R}$ mice, suggesting that apoptosis was not the major cause for the phenotypes (Supplementary Fig. 2c). However, when we treated bone marrow-derived macrophages (BMDMs) from the Tnfr1 $1^{-/-}$Ripk $1^{K 376 R / K 376 R}$ mice with LPS or Poly(I:C) in the presence of $\mathrm{zVAD}$, the cells were much more vulnerable compared to their controls and the phosphorylations of RIPK1 and RIPK3 were more significantly induced in these cells, suggesting that RIPK3-mediated necroptosis plays an important role in this circumstance (Supplementary Fig. 2f, g). Therefore, we tested the contribution of RIPK3-mediated signaling by crossing Ripk $3^{-/-}$into Tnfr1 $1^{-/-}$Ripk1 $1^{\text {K376R/K376R }}$ mice. Importantly, deletion of Ripk 3 rescued the postnatal death of Tnfr1 $1^{-1-}$ Ripk $1^{\text {K376R/K376R }}$ and allowed them normal development (Fig. 6f, h). Moreover, histological examination indicated that Ripk3 ablation markedly prevented the tissue loss and inflammation in Tnfr $1^{-/-}$Ripk $1^{K 376 R / K 376 R}$ mice (Fig. 6g). Taken together, these findings reveal a critical role of K376-meidated RIPK1 ubiquitination in restraining RIPK3-dependent inflammation during postnatal development.

Ablation of Fadd and Ripk3/Mlkl rescues Ripk1 ${ }^{\text {K376R/K376R }}$ mice. Given that the activation of RIPK1 led to two subsequent signaling pathways including FADD/Caspase-8-mediated apoptosis and RIPK3/MLKL-mediated necroptosis, we next asked whether $R i p k 1^{K 376 R / K 376 R}$ lethality was caused by these pathways. First, we crossed Ripk $1^{K 376 R /+}$ into Ripk $3^{-/-}$or $M l k l^{-/-}$mice to test the contribution of necroptosis. The resulting Ripk3 ${ }^{-/}$Ripk $1^{K 376 R / K 376 R}$ and $M l k l^{-/-}$Ripk $1^{K 376 R / K 376 R}$ mice died during embryogenesis as we observed no homozygous descendant from heterozygous breeding (Supplementary Fig. 3b). The results from timed preg-

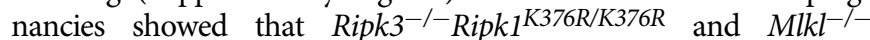
$R i p k 1^{K 376 R / K 376 R}$ embryos appeared normal at E13.5 but displayed apparent developmental abnormalities at E14.5 (Supplementary Fig. 3a). Although ablation of Ripk3 or Mlkl slightly prolonged the survival of Ripk1 K376R/K376R embryos, the lethality was not prevented. These findings indicate the involvement of RIPK3/MLKLindependent pathways.

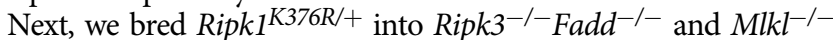
Fadd ${ }^{-1-}$ mice to generate Ripk3 ${ }^{-/}$Fadd ${ }^{-/}{ }^{-}$Ripk1 ${ }^{\text {K376R/K376R }}$ and $\mathrm{Mlkl}^{-1-}$ Fadd $^{-1-}$ Ripk1 ${ }^{\text {K376R/K376R }}$ mice, respectively. Ablation of Fadd and Ripk3 or Mlkl fully rescued the embryonic lethality of Ripk1 $1^{K 376 R / K 376 R}$ mice and displayed normal development and maturation (Fig. 7a, b). MEFs isolated from these triple mutant mice were resistant to TNF- $\alpha$ triggered apoptosis and necroptosis while were defective in activating NF- $\mathrm{KB}$ signaling as $R i p k 1^{K 376 R / K 376 R}$ cells, suggesting that massive cell death rather than impaired NF- $\kappa B$ activation contributes to the lethality of Ripk $1^{K 376 R / K 376 R}$ mice (Supplementary Fig. 4b, c). RIPK1 ${ }^{\mathrm{K} 376 \mathrm{R}}$ was widely detected in the 
a

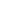

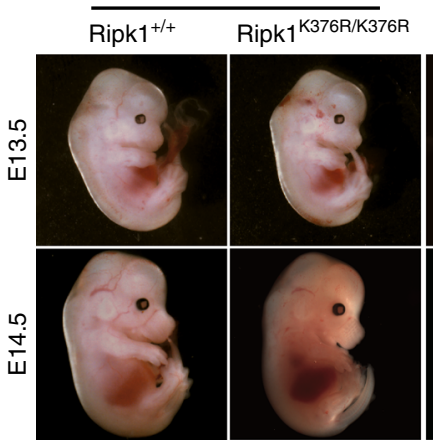

C

\begin{tabular}{|c|c|c|}
\hline $\begin{array}{l}\text { Tnfr1 } 1^{-/-} \text {Ripk } 1^{\text {K376R/+ }} \\
\times \text { Tnfr }^{-/-} \text {Ripk1 } 1^{\text {K376R/+ }}\end{array}$ & Observed & Expected \\
\hline Tnfr $1^{-/-} \mathrm{Ripk}^{+/+}$ & 13 & 14 \\
\hline Tnfr1 ${ }^{-/-}$Ripk $1^{\text {K376R/+ }}$ & 33 & 28 \\
\hline Tnfr1 $^{-/-}$Ripk1 ${ }^{\text {K376R/K376R }}$ & 10 & 14 \\
\hline Total & 56 & 56 \\
\hline
\end{tabular}

e
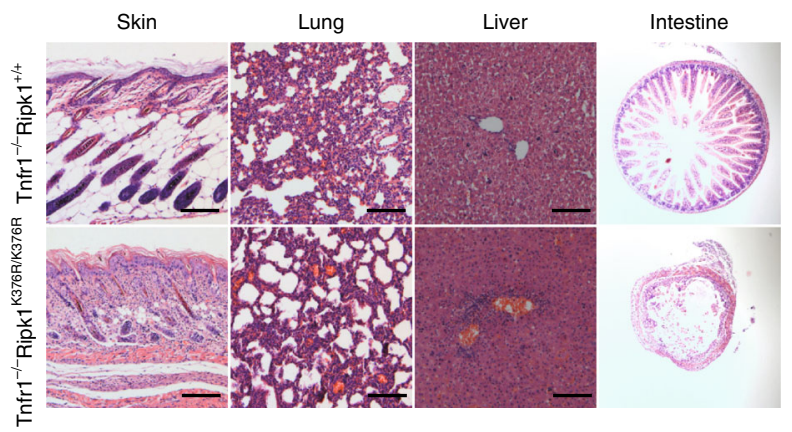

g

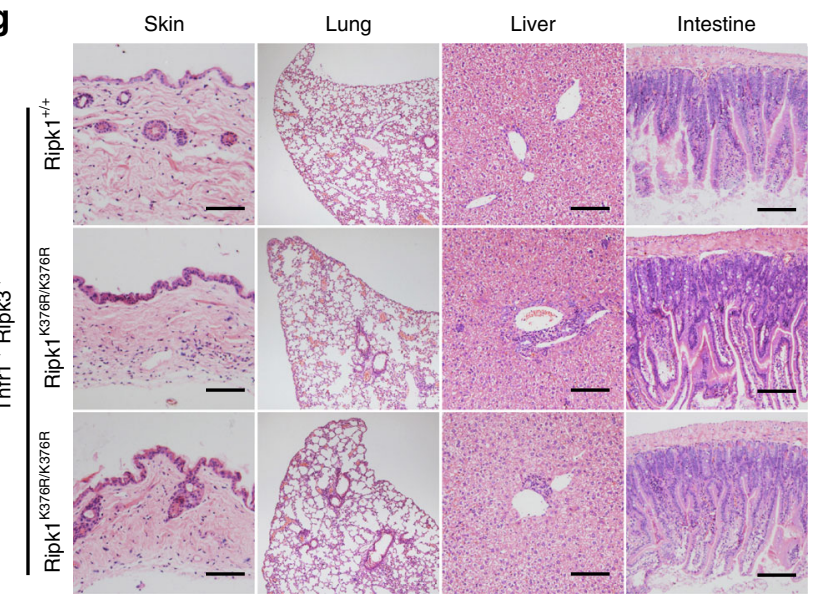

b
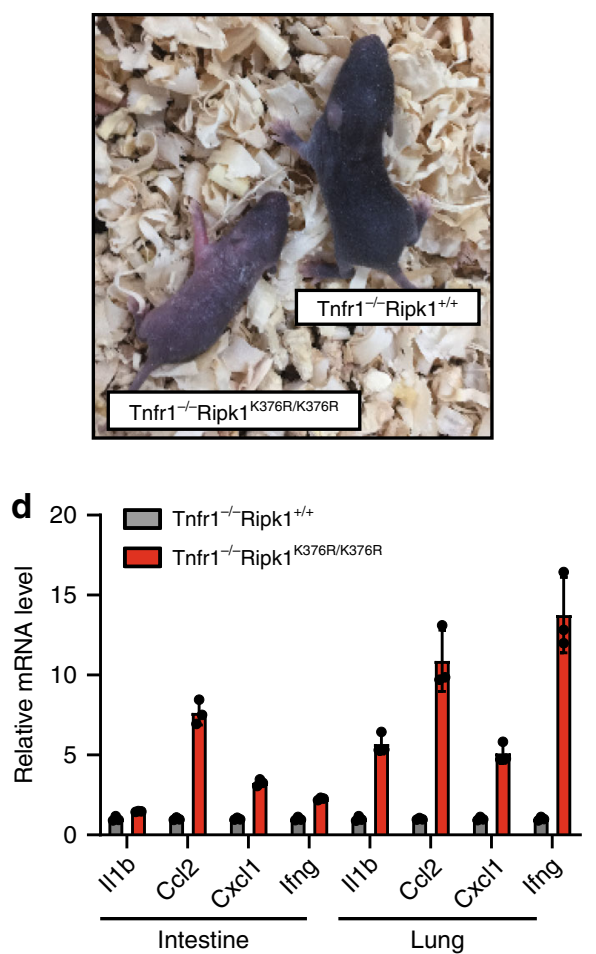

f

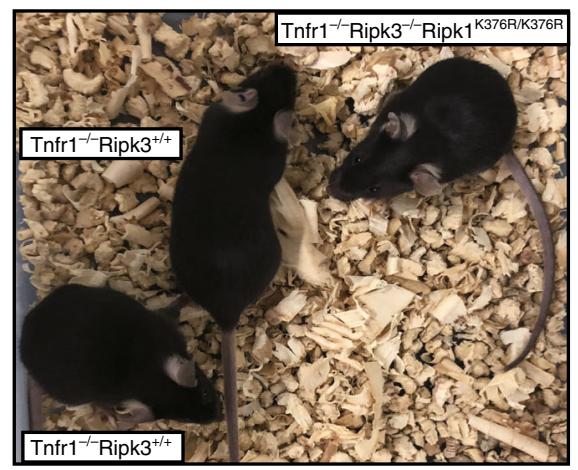

h

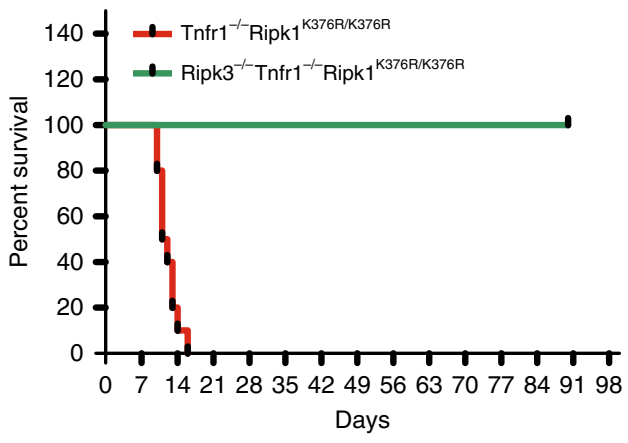

major organs of triple-mutant mice and its expression levels were comparable to those of WT controls, suggesting that RIPK1 ${ }^{\mathrm{K} 376 \mathrm{R}}$ did not affect the expression of RIPK1 (Supplementary Fig. 4a).

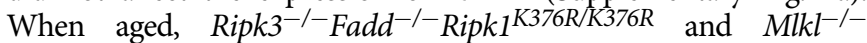
$\mathrm{Fadd}^{-1-}$ Ripk1 ${ }^{\text {K376R/K376R }}$ mice developed lymphoproliferative syndrome with an accumulation of $\mathrm{CD}^{+} \mathrm{B} 220^{+}$lymphocytes in peripheral lymphoid organs seen in Ripk3 ${ }^{-/} \mathrm{Fadd}^{-/-}$and $M_{l k l^{-/}}$Fadd $^{-1-}$ mice (Fig. 7d, f, Supplementary Fig. 4d) ${ }^{19-22,41-43}$. The disease was characterized by lymphadenopathy and splenomegaly, which disrupted the normal architecture of the lymph nodes and spleen (Fig. 7c, d). Flow cytometric analysis revealed profoundly increased numbers of multiple cells in spleen and lymph nodes, 
Fig. 6 Tnfr1-/-Ripk $7^{K 376 R / K 376 R}$ mice die postnatally due to RIPK3-dependent inflammation. a Whole-mount dark field images of embryos with indicated

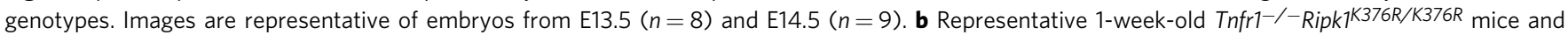
littermate control. c Expected and observed frequency of genotypes in offspring at weaning from crosses of Tnfr1-/-Ripk $7^{K 376 R /+}$. d Quantitative RT-PCR analysis of the mRNA expression of cytokines in lungs and intestines from 1-week-old pups with indicated genotypes. Data are represented as mean \pm SEM $(n=3)$. Source data are provided as a Source Data file. e Representative H\&E stained tissues from 1-week-old Tnfr1 ${ }^{-/}-$Ripk $7^{K 376 R / K 376 R}$ and littermate control. Scale bars, $100 \mu \mathrm{m}$. f Representative 8-week-old Tnfr1-/-Ripk3-/-Ripk 7 K376R/K376R and its littermate controls. $\mathbf{g}$ Representative H\&E stained tissues from 8-week-old Tnfr1-/-Ripk3-/-Ripk $7^{K 376 R / K 376 R}$ and littermate control. Scale bars, $100 \mu \mathrm{m}$. h Kaplan-Meier plot of mouse survival from intercrosses of $\operatorname{Tnfr}^{-/-}$Ripk $7^{K 376 R /+}$ Ripk3 $3^{+/-}$

including lymphocytes ( $\mathrm{T}$ cells/B cells), macrophages and granulocytes (Fig. 7e). In addition, the inflammation was observed

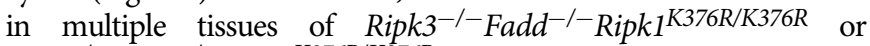
$M l k l^{-1-}$ Fadd ${ }^{-1-}$ Ripk $1^{K 376 R / K 376 R}$ mice, indicating the occurrence of systemic inflammation (Fig. 7c). Collectively, these data indicate that blockade of both FADD-mediated apoptotic pathway and RIPK3/MLKL-mediated necroptotic pathway rescues the embryonic lethality of Ripk $1^{K 376 R / K 376 R}$ mice, although these mice develop lymphoproliferative diseases with systemic inflammation.

\section{Discussion}

In this report, we show that the disruption of RIPK1 ubiquitination in vivo by a point mutation at K376 causes embryonic lethality at E12.5, which is completely different from the observation in Ripk1 deficient mice. A fundamental question emerged from this result: why does germline Ripk $1^{K 376 R}$ mutation cause lethality during embryogenesis while deletion of Ripk1 does not? The answer to the question may come from the unique structure of RIPK1 which contains a C-terminal death domain (DD), an intermediate domain harboring a RHIM and an N-terminal Ser/ Thr kinase domain. The DD and RHIM can, respectively, recruit FADD and RIPK3 to initiate extrinsic apoptosis and necroptosis, while the intermediate domain can mediate the activation of NF$\kappa \mathrm{B}$ signaling $28,44,45$. In our study, we substituted K376 of the intermediate domain with R376 to block polyubiquitination of RIPK1. This mutation leads to two consequences: the defective NF- $\kappa \mathrm{B}$ activation and the excessive cell death. These findings allowed two conclusions: (1) K376-mediated RIPK1 ubiquitination is responsible to activate NF- $\mathrm{KB}$ in vivo and (2) K376mediated RIPK1 ubiquitination is critical to suppress apoptosis and necroptosis during development. In fact, RIPK1 ${ }^{\mathrm{K} 376 \mathrm{R}}$ mutation does not affect the expression of RIPK1 and functions of other domains, but was prone to the activation of RIPK1. This can be prevented by RIPK1 kinase inhibitor Nec-1 or deletion of one Ripk1 ${ }^{K 376 R}$ allele, indicating that the excessive RIPK1 activation which subsequently causes the apoptosis and necroptosis leads to the lethality during embryogenesis. Therefore, RIPK1 ubiquitination restrains RIPK1 itself from activating cell death during embryonic development, and that's why deletion of Ripk1 does not lead to lethal phenotypes at this stage.

Ripk $1^{-/-}$mice die soon after birth, this lethality is initially thought to be due to the loss of the pro-survival function of RIPK1 which mediates NF- $\mathrm{KB}$ signaling ${ }^{46}$. The contribution of NF- $\kappa B$ signaling to maintain normal embryonic development has been investigated previously, and mice lacking the components of $\mathrm{NF}-\mathrm{\kappa B}$ die during embryogenesis due to massive cell death in multiple tissues ${ }^{47-49}$. Moreover, inactivating RIPK1 kinase can rescue the embryonic lethality of RelA-deficient mice ${ }^{50,51}$. The phenotypes of these mice resemble what we observed in Ripk1 ${ }^{K 376 R / K 376 R}$ mice, indicating that impaired NF- $\mathrm{KB}$ signaling may be a cause that leads to the embryonic lethality of Ripk1 ${ }^{K 376 R / K 376 R}$ mice. However, when treating Ripk $1^{\text {K376R/K376R }}$ cells with TNF- $\alpha$ combined with CHX which blocked NF-kBdependent gene expression, Ripk $1^{K 376 R / K 376 R}$ MEFs were still more vulnerable to the stimuli. Furthermore, activating NF- $\mathrm{kB}$ signaling by $\mathrm{p} 65^{\mathrm{S} 275 \mathrm{D}}$ overexpression failed to prevent the cell death of mutant cells. Besides, it has been reported that RIPK1 K377-mediated (human RIPK1) inhibition of TNF- $\alpha$ induced apoptosis is independent on NF- $\mathrm{kB}$ at the early stage of stimulation ${ }^{38}$, which is consistent with what we observed in Ripk1 ${ }^{\text {K376R/K376R }}$ cells. Therefore, NF-kB defects might not be the leading cause of Ripk1 ${ }^{K 376 R / K 376 R}$ lethality.

The perinatal death of Ripk $1^{-/-}$mice was fully rescued by ablation of both Caspase-8/Fadd and Ripk $3^{19-22}$. Thus, RIPK1 was considered to suppress lethal pathways engaged by FADDCaspase-8 and RIPK3. However, the mechanism by which RIPK1 suppresses the perinatal lethality remains elusive. The mutation of RHIM in RIPK1 causes perinatal death similar to the observations from Ripk1-1- mice, and this lethality can be rescued by ablation of Ripk3 or Zbp $1^{35,36}$. These investigations suggest that RIPK1 suppresses the perinatal necroptosis mediated by RIPK3/ZBP1 through RHIM domain. In our study, ablation of Ripk3 rescues the postnatal death of Tnfr $1^{-/-}$Ripk $^{\text {K376R/K376R }}$ mice. Thus, we assume that RIPK1 K376 is important for restraining RIPK3-dependent necroptosis and ensuing inflammation after birth. Intriguingly, in the settings of RIPK1 $1376 \mathrm{R}$ mutation, RIPK1 fails to prevent the postnatal necroptosis through RHIM, suggesting that K376-mediated ubiquitination may regulate RHIM-mediated suppression of RIPK3-dependent necroptosis.

Although ablation of Tnfr1 rescued Ripk1 ${ }^{K 376 R / K 376 R}$ mice during embryonic development, Tnfr $1^{-/}$Ripk $1^{\text {K376R/K376R mice }}$ died postnatally. These results indicate RIPK1 ubiquitination on K376 may mediate divergent pathways at different stages of development. TNF/TNFR1 is the main upstream signal of K376mediated pathways during embryonic development, while additional signals are involved after birth. Although it is not clear which signaling pathways participated, the investigations on Ripk $1^{-/-}$mice could provide some candidates. Similar to our

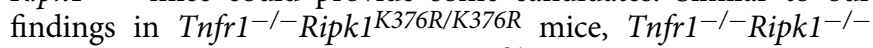
mice died within 2 weeks after birth ${ }^{21}$. Interestingly, deletion of Trif or type I interferon receptor prolonged the lifespan of Ripk $1^{-/-}$Tnfr $1^{-/-}$mice, although none of the mice survived past weaning ${ }^{21}$. In addition, deletion of MyD88 improved the survival time of Ripk1 $1^{-/-}$mice ${ }^{22}$. Therefore, we assume that, in addition to TNFR1, IFNR1, and TRIF are of great possibility to trigger the RIPK1 K376-meidated pathways. Intriguingly, as ablation of Ripk3 rescues the lethality of $\operatorname{Tnfr} 1^{-/}$Ripk1K376R/K376R mice, the downstream signaling mediating the lethal phenotypes is dependent on RIPK3. Recent studies have implied that RIPK3 has more functions beyond mediating necroptosis, therefore, additional investigations on examining the contribution of MLKL in this signaling pathway are still required.

By changing its ubiquitination status in response to TNF- $\alpha$, RIPK1 can transduce either a pro-survival or death signal. Germline mutation of RIPK1 K376 leads to embryonic lethality at E12.5, which was prevented by deletion of Tnfr1, though Tnfr1 ${ }^{-1-}$ Ripk1 ${ }^{\text {K376R/K376R }}$ mice died of RIPK3-mediated inflammation after birth. Remarkably, one copy of RIPK1 is 
a

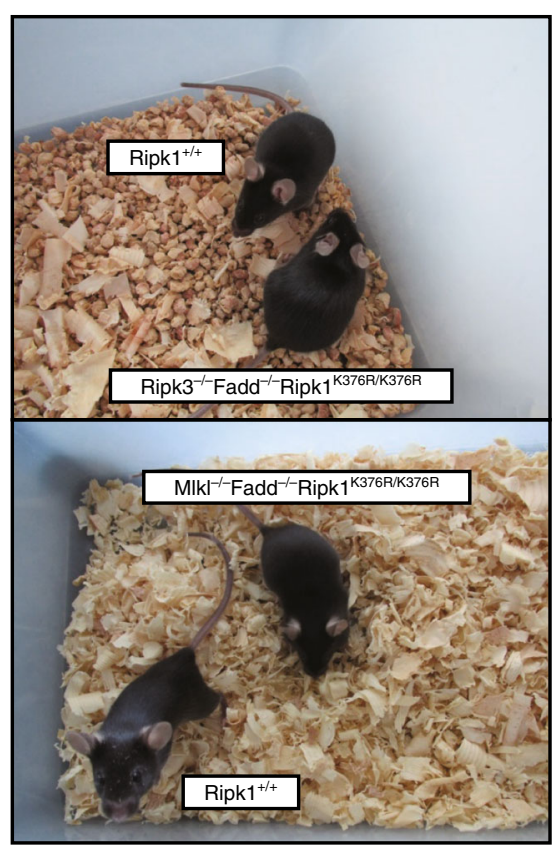

c

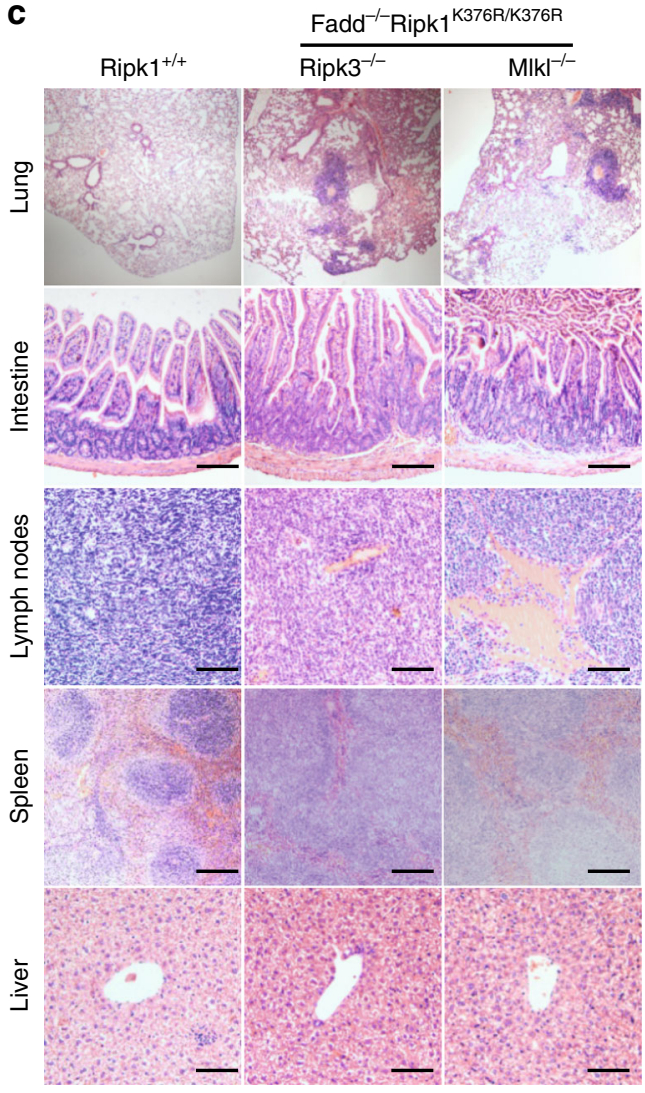

b

\begin{tabular}{|c|c|c|}
\hline $\begin{aligned} & \text { Ripk3 }^{-/-} \text {Fadd }^{-/} \text {Ripk1 } 1 \\
\times & \text { Ripk3 }^{\text {K37-FR/+ }} \text { Fadd }^{-/} \text {Ripk1 }^{\text {K376R/+ }}\end{aligned}$ & Expected & Observed \\
\hline Ripk3 $^{-/-}$Fadd $^{+/-}$Ripk $^{+/+}$ & 9 & 11 \\
\hline Ripk3 $^{-/-}$Fadd $^{+/-}$Ripk1 $1^{\text {K376R/+ }}$ & 18 & 20 \\
\hline Ripk3 $^{-/-}$Fadd $^{+/-}$Ripk1 $^{\text {K376R/K376R }}$ & 9 & 0 \\
\hline Ripk3 $^{-/-}$Fadd $^{-1-}$ Ripk $^{+/+}$ & 9 & 12 \\
\hline Ripk3 $^{-/-}$Fadd $^{-/-}$Ripk1 ${ }^{\text {K376R/+ }}$ & 18 & 21 \\
\hline Ripk3 $^{-/-}$Fadd $^{-/-}$Ripk1 $1^{\text {K376R/K376R }}$ & 9 & 8 \\
\hline Total & 72 & 72 \\
\hline $\begin{aligned} & \text { Mlkl }^{-/-} \text {Fadd }^{-/-} \text {Ripk } 1^{13376 R /+} \\
\times & \text { Mlkl }^{1 /-} \text { Fadd }^{+/-} \text {Ripk } 1^{K 376 R /+}\end{aligned}$ & Expected & Observed \\
\hline $\mathrm{MlkI}^{-/-}$Fadd $^{+/-}$Ripk $1^{+/+}$ & 8 & 10 \\
\hline $\mathrm{Mlkl}^{-/-}$Fadd $^{+/-} \operatorname{Ripk}^{\mathrm{K} 376 \mathrm{R} /+}$ & 16 & 18 \\
\hline $\mathrm{Mlkl}^{-/-}$Fadd $^{+/-}$Ripk1 $1^{\mathrm{K} 376 \mathrm{R} / \mathrm{K} 376 \mathrm{R}}$ & 8 & 0 \\
\hline $\mathrm{Mlkl}^{-/-}$Fadd $^{-/-} \mathrm{Ripk}^{+/+}$ & 8 & 10 \\
\hline Mlkl $^{-/-}$Fadd $^{-/-}$Ripk1 $1^{\text {K376R/+ }}$ & 16 & 19 \\
\hline $\mathrm{Mlkl}^{-1-} \mathrm{Fadd}^{-1-} \mathrm{Ripk}^{\mathrm{K} 376 \mathrm{R} / \mathrm{K} 376 \mathrm{R}}$ & 8 & 7 \\
\hline Total & 64 & 64 \\
\hline
\end{tabular}

d
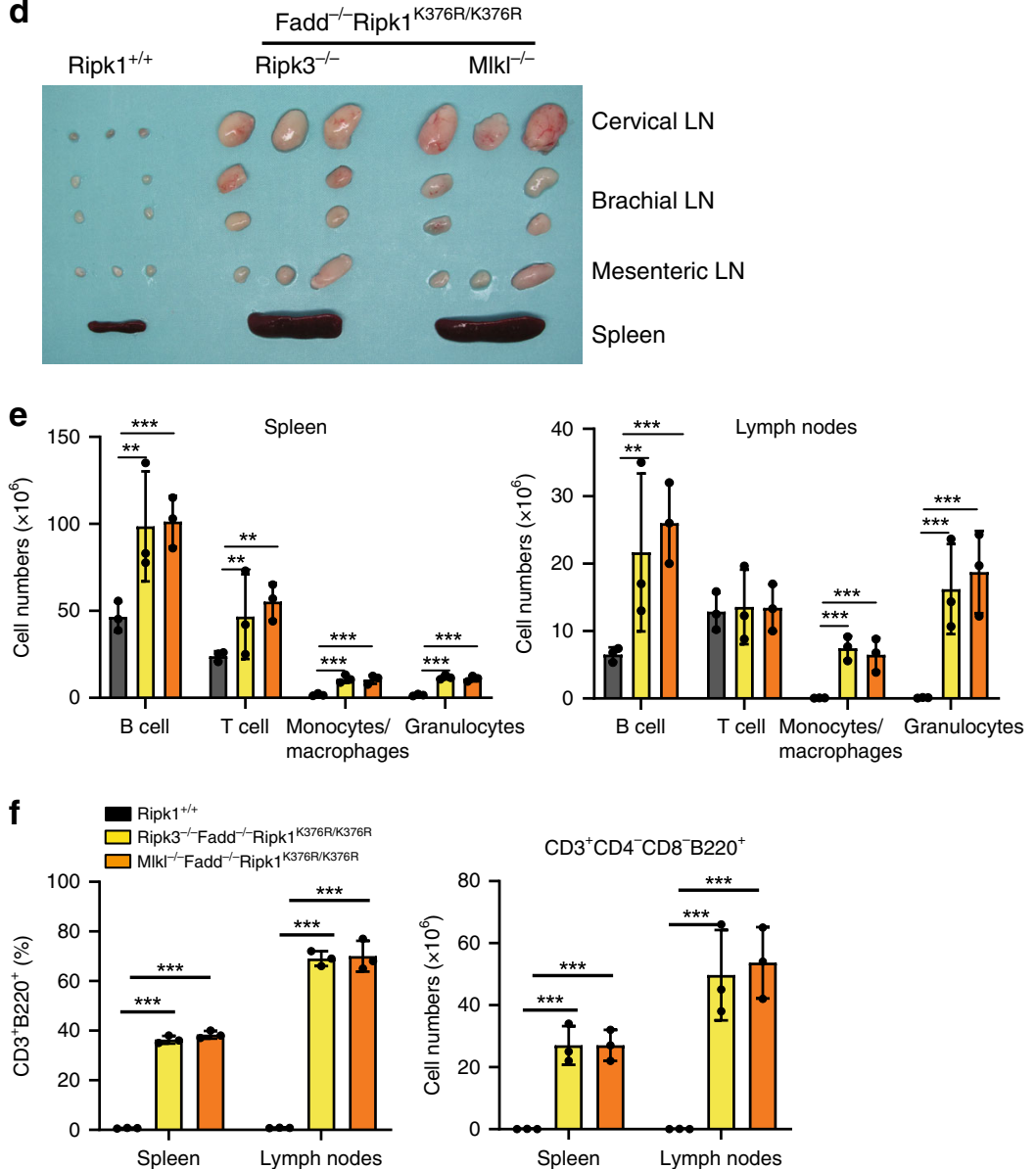

required to prevent a Fadd/Ripk3 or Fadd/Mlkl dependent lethality in Ripk1 $1^{K 376 R / K 376 R}$ mice, possibly relating to a RIPK1 activation threshold (Supplementary Fig. 5). These genetic evidences provide a comprehensive understanding of the in vivo function of RIPK1 ubiquitination, which is essential for both embryonic development and immune homeostasis.
Importantly, these findings can help to explain some seemingly contradictory results from previously reported genetic studies. Furthermore, a more recent study has highlighted the role of RIPK1 in human disease asscoiated with immunodeficiency, arthritis, and intestinal inflammation, which confirmed the function of RIPK1 in humans immune system ${ }^{52}$. Therefore, the 
Fig. 7 Embryonic lethality of Ripk $7376 R / K 376 R$ mice is fully rescued by ablation of Fadd and Ripk3/Mlkl. a Representative photographs of mice with the indicated genotypes. b Predicted and observed frequencies in offspring from crosses of animals with the indicated genotypes. c Representative H\&Estained tissues from 12-week-old animals of the indicated genotypes. Results are representative of three mice of each genotype (Scale bars, $100 \mu \mathrm{m}$ ). $\mathbf{d}$ Representative lymphoid organs removed from 12-week-old mice of indicated genotypes. e Different cell subsets from 12-week-old animals were determined by flow cytometry using following markers: B cells $\left(\mathrm{CD} 19^{+}\right.$or $\left.\mathrm{B} 22 \mathrm{O}^{+}\right)$, $\mathrm{T}$ cells $\left(\mathrm{CD} 3^{+}\right)$, Monocytes and macrophages $\left(\mathrm{CD} 11 \mathrm{~b}^{+}\right)$, Granulocytes $\left(\mathrm{Gr}-1^{+}\right)$. Data are represented as mean \pm SEM $\left(n=3\right.$ /genotype). $P$ values were determined by Student's $t$-test $\left({ }^{\star \star} p<0.01,{ }^{\star \star \star} p<0.001\right)$. f Percentages and cell numbers of CD3+B220+ lymphocytes in spleen and lymph nodes from 12-week-old mice of indicated genotypes. Data are represented as mean \pm SEM $\left(n=3\right.$ /genotype). $P$ values were determined by Student's $t$-test $\left({ }^{\star \star} p<0.01,{ }^{\star \star \star} p<0.001\right)$. Source data are provided as a Source Data file

potential role of RIPK1 ubiquitination in regulating specific signaling pathways other than the TNF-a mediated pathway will be of great interest for providing new therapeutic opportunities in related human diseases, and mice bearing RIPK1 ${ }^{\mathrm{K} 376 \mathrm{R}}$ mutation can be used to explore the contribution of RIPK1 ubiquitination to a variety of mouse models of human diseases.

\begin{abstract}
Methods
Mice. Mice were housed in a specific pathogen-free (SPF) facility. Ripk $3^{-/-}$, $\mathrm{Fadd}^{+/-}$and $\mathrm{Mlkl}^{-/-}$, and Ifnar ${ }^{-/-}$mouse lines have been described earlier ${ }^{41,53}$ Ripk1 ${ }^{K 376 R /+}$ and Ripk1 $1^{+/-}$mice were generated by CRISPR-Cas9 mutation system (Bioray Laboratories Inc., Shanghai, China). The mouse Ripk $1^{K 376 R}$ mutation construct corresponds to the following genomic position (NC_000079.6) chr13: 34027834-34027836. Ripk1 ${ }^{\text {K376R/+ }}$ mouse genotyping primers: $5^{\prime}$ CCATCTCCA GCCCTCCTA and 5' TGCACTGCAATTCCACGA amplified 690 bp DNA fragments for sequencing. Ripk $1^{+/-}$mice were generated by a $101 \mathrm{bp}$ deletion in the coding region of exon 2 using CRISPR-Cas9 mutation system with sgRNA ( $5^{\prime}-$ GACCTAGACAGCGGAGGCTT-3'). Ripk $1^{+/-}$mice genotyping primers (RIPK1-

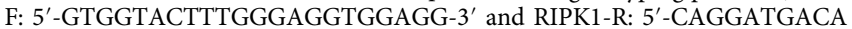
AATCCATGGCTTCTG-3') amplified 430 bp wild-type, 329 bp knock-out DNA fragments. Additional information is provided upon request. All mice utilized in this study have been backcrossed onto the C57BL/ 6 background for more than eight generations. Animal experiments were conducted in accordance with the guidelines of the Institutional Animal Care and Use Committee of the Institute of Nutrition and Health, Shanghai Institutes for Biological Sciences, University of
\end{abstract} Chinese Academy of Sciences.

Reagents. The Smac mimetic, Cycloheximide and LPS were purchased from Sigma. TNF- $\alpha$ and $z-V A D$ were from R\&D (Minneapolis, MN, USA) and Calbiochem (Anaheim, CA, USA), respectively. Necrostatin-1 was purchased from Enzo Life Science (Alexis, USA). The following antibodies were used for western blotting and immunoprecipitation experiments: RIPK1 (1:2000, BD Biosciences, 610459), RIPK1 (1:2000, Cell Signaling Technology, 3493P), p-RIPK1(1:1000, a gift from Junying Yuan's lab), RIPK3 (1:5000, Prosci, 2283), p-RIPK3(1:1000. Abcam, ab195117), Caspase-8 (1:2000, Enzo Life Science, ALX-804-447-C100), MLKL (1:2000, Abgent, ap14272b), p-MLKL (1:1000, Abcam, ab196436), ß-actin (1:5000, Sigma, A3854), Tublin (1:5000, Sigma, T6199), IkBa (1:2000, Cell Signaling Technology, 9242S), p-IkBa (1:1000, Cell Signaling Technology, 9246S), p-ERK (1:2000, Cell Signaling Technology, 9101S), ERK (1:2000. Cell Signaling Technology,9102S), p-p38 (1:2000, Cell Signaling Technology, 9211S), p38 (1:2000, Cell Signaling Technology, 9228S), p-p65 (1:2000, Cell Signaling Technology, 3033S), p65 (1:2000, Cell Signaling Technology, 4764S), p-JNK (1:1000, Cell Signaling Technology, 9251S), JNK (1:2000, Cell Signaling Technology, 9252S), PARP (1:2000,Cell Signaling Technology, 9542S), Caspase-3 (1:1000,Cell Signaling Technology, 9662S), TBP (1:2000,Cell Signaling Technology, 44059S), FADD antibody was from Jianke Zhang's lab (1:2000, Thomas Jefferson University). Cell viability was determined by measuring ATP levels using the Cell Titer-Glo kit (Promega).

Cell lines. Mouse embryonic fibroblasts (MEFs) were cultured in Dulbecco's modified Eagle's medium (Hyclone, SH30243.LS) supplemented with 10\% fetal bovine serum (Hyclone, SH30084.03),1\% penicillin $(100 \mathrm{IU} / \mathrm{ml}) /$ streptomycin $(100 \mu \mathrm{g} / \mathrm{ml})$ and $2 \mathrm{mM} \mathrm{L}$-glutamine under $5 \% \mathrm{CO} 2$ at $37^{\circ} \mathrm{C}$. Primary Ripk $1^{+/+}$and Ripk $1^{K 376 R / K 376 R}$ MEFs were isolated from E11.5 littermate embryos. The head and visceral tissues were dissected and remaining bodies were incubated with $4 \mathrm{ml}$ Tripsin/EDTA solution (Invitrogen, 25200072) per embryo at $37^{\circ} \mathrm{C}$ for $1 \mathrm{~h}$. After tripsinization, an equal amount of medium were mixed and pipetted up and down a few times. The primary MEFs were cultured in high-glucose DMEM containing $15 \%$ FBS. For immortalization, MEFs were transfected with SV40 small+large T antigen-expressing plasmid (Addgene, 22298) using Lipofectamine 2000 (Invitrogen, 11668019) according to manufacturer's instructions.
Cell survival assay. Cell survival was determined using the CellTiter-Glo Luminescent Cell Viability Assay kit (Promega, G7572) and the luminescence was recorded with a microplate luminometer (Thermo Scientific).

Immunoblotting and immunoprecipitation. Cells were harvested at different time points, washed with PBS and lysed with $1 \times$ SDS sample buffer containing $100 \mathrm{mM}$ DTT. The cell lysates were cleared by centrifugation for $10 \mathrm{~min}$ at $12,000 \times g$, quantified by BCA kit (Thermo Scientific,23225), and then mixed with SDS sample buffer and boiled at $95^{\circ} \mathrm{C}$ for $5 \mathrm{~min}$. The samples were separated using SDS-PAGE, transferred to PVDF membrane (Millipore) with $110 \mathrm{v}$ for $1.5 \mathrm{~h}$. The proteins were detected by using a chemiluminescent substrate (Thermo Scientific). To immunoprecipitate RIP1, cells were lysed with lysis buffer (Tris-HCl $20 \mathrm{mM}$ (pH 7.5), $\mathrm{NaCl} 150 \mathrm{mM}$, EDTA 1 mM, EGTA 1 mM, Triton X-100 1\%, Glycerol 10\%, Protease Inhibitor cocktail, Sodium pyrophosphate $2.5 \mathrm{mM}, \beta$-Glycerrophosphate $1 \mathrm{mM}, \mathrm{NaVO}_{4} 1 \mathrm{mM}$, Leupeptin $\left.1 \mu \mathrm{g} / \mathrm{ml}\right)$. Cell lysates were incubated for $3 \mathrm{~h}$ with $1 \mu \mathrm{g}$ of RIP1 antibody (BD Biosciences, 610459). After mixing end over end for overnight $\left(4^{\circ} \mathrm{C}\right)$ with $35 \mu \mathrm{l}$ of $\mathrm{G}$-Agarose beads, the agarose was collected and washed three times with lysis buffer. Immunoprecipitates were denatured in SDS, subjected to SDS-PAGE, and immunoblotted. To immunoprecipitate Complex I, cells were harvest in NP-40 buffer (Tris- $\mathrm{HCl} 20 \mathrm{mM}$ (pH 7.5), $\mathrm{NaCl} 120 \mathrm{mM}$, EDTA 1 mM, EGTA 1 mM, NP-40 0.5\%, Glycerol 10\%, Protease Inhibitor cocktail, Sodium pyrophosphate $2.5 \mathrm{mM}, \beta$-Glycerrophosphate $1 \mathrm{mM}, \mathrm{NaVO}_{4} 1 \mathrm{mM}$, Leupeptin $1 \mu \mathrm{g} / \mathrm{ml}$ ) after stimulating with $100 \mathrm{ng} / \mathrm{ml}$ TNF- $\alpha$-FLAG(Enzo Life Science, ALX-522-009-C050). The cell lysates were then incubated with FLAG-tagged beads (Sigma, A2220) for $4 \mathrm{~h}$. The beads were then collected and washed 3 times with NP-40 buffer. Immunoprecipitates were denatured in SDS, subjected to SDSPAGE, and immunoblotted. The unprocessed scans of all blots are provided as Supplementary Fig. 6 in the Supplementary Information.

MLKL oligomerization detection. Cells were harvested after TSZ or TSZN treatment and lysed with $2 \times$ DTT-free sample buffer (Tris-Cl (PH 6.8) $125 \mathrm{mM}$, SDS $4 \%$, Glycerol 20\%, Bromophenol blue 0.02\%) immediately. Total cell lysates were separated using SDS-PAGE, and transferred to PVDF membrane, and immunoblotting was performed with MLKL antibodies.

Flow cytometry. Lymphocytes were isolated from the thymus, spleen, and lymph nodes of mice. Antibodies against mouse CD3 (1:1000, eBioscience,11-0031-82), CD4 (1:1000, eBioscience,47-0042-82), CD8 (1:1000,Biolegend,100732), B220 (1:1000, eBioscience,12-0452-83), Gr-1 (1:1000, eBioscience, 12-5931-83), CD11b (1:1000, eBioscience, 17-0112-83) were used for flow cytometry analysis in this study. Single cell suspension of lymphocytes was stained on ice for half an hour with fluorescence-conjugated antibodies in the staining buffer $(1 \times \mathrm{PBS}, 3 \% \mathrm{BSA}, 1$ mM EDTA, $0.1 \% \mathrm{NaN} 3$ ). After staining, cells were washed twice with PBS and immediately analyzed by flow cytometry (FACS Aria III, BD biosciences).

RT-PCR. Total RNA was extracted using Trizol reagent (Life Technologies), according to the manufacturer's instructions. After quantification, $1 \mu \mathrm{g}$ total RNA was reverse transcribed to complementary DNA (Takara). Transcript levels of indicated genes were quantified by quantitative RT-PCR on an ABI 7500 real-time PCR instrument with SYBR Green. The sequences of primers are shown as follows. Mcp-1: 5'-AGGTGTCCCAAAGAAGCTGTA-3' and 5'-ATGTCTGGACCCATTC CTTCT-3'; A20: 5'-CTCAGAACCAGAGATTCCATGAAG-3' and 5'-CCTGTGTA GTTCGAGGCATGT-3'; Icam-1: 5'-CTTGGTAGAGGTGACTGA-3' and 5'- GCT GAAAAGTTGTAGACTG-3'; I $\kappa \alpha$ : 5'-GATCCGCCAGGTGAAGGG-3' and 5'GCAATTTCTGGCTGGTTGG-3'; Irf1: 5'-CAGAGGAAAGAGAGAAAGTCC-3' and 5'-CACACGGTGACAGTGCTGG-3'; $I l-1 b$ : 5'- CCCAACTGGTACATCAG CAC-3' and 5'-TCTGCTCATTCACGAAAAGG-3'; Ccl2: 5'-TGAATGTGAAGTT GACCCGT-3' and 5'-AAGGCATCACAGTCCGAGTC-3'; Cxcl1: 5'-CAAGAACA TCCAGAGCTTGAAGGT-3' and 5'-GTGGCTATGACTTCGGTTTGG-3'; Ifng: 5' ACAGCAAGGCGAAAAAGGAT-3' and 5'-TGAGCTCATTGAATGCTTGG-3';

Statistical analysis. Data presented in this article are representative results of at least three independent experiments. The statistical significance of data was evaluated by Student's $t$ test and the statistical calculations were performed with GraphPad Prism software. 
Reporting summary. Further information on research design is available in the Nature Research Reporting Summary linked to this article.

\section{Data availability}

The authors declare that all data presented in this study are available within the figures and its Supplementary Information file. The source data underlying Figs. 3a, 4a, e, 5b, f, g, 6d, 7e, f and Supplementary Figs 1b, 2e, f are provided as a Source Data file. Other data that support the study are available from the corresponding author upon reasonable request.

Received: 19 December 2018 Accepted: 30 July 2019

Published online: 13 September 2019

\section{References}

1. Micheau, O. \& Tschopp, J. Induction of TNF receptor I-mediated apoptosis via two sequential signaling complexes. Cell 114, 181-190 (2003).

2. Chen, G. \& Goeddel, D. V. TNF-R1 signaling: a beautiful pathway. Science 296, 1634-1635 (2002).

3. Ea, C. K., Deng, L., Xia, Z. P., Pineda, G. \& Chen, Z. J. Activation of IKK by TNFalpha requires site-specific ubiquitination of RIP1 and polyubiquitin binding by NEMO. Mol. Cell 22, 245-257 (2006).

4. Kanayama, A. et al. TAB2 and TAB3 activate the NF-kappaB pathway through binding to polyubiquitin chains. Mol. Cell 15, 535-548 (2004).

5. Li, H. X., Kobayashi, M., Blonska, M., You, Y. \& Lin, X. Ubiquitination of RIP is required for tumor necrosis factor alpha-induced NF-kappa B activation. J. Biol. Chem. 281, 13636-13643 (2006).

6. Wang, C. et al. TAK1 is a ubiquitin-dependent kinase of MKK and IKK. Nature 412, 346-351 (2001).

7. Bertrand, M. J. M. et al. cIAP1 and cIAP2 facilitate cancer cell survival by functioning as E3 ligases that promote RIP1 ubiquitination. Mol. Cell 30, 689-700 (2008).

8. Geserick, P. et al. Cellular IAPs inhibit a cryptic CD95-induced cell death by limiting RIP1 kinase recruitment. J. Cell Biol. 187, 1037-1054 (2009).

9. Kovalenko, A. et al. The tumour suppressor CYLD negatively regulates NFkappa B signalling by deubiquitination. Nature 424, 801-805 (2003).

10. Wang, L., Du, F. H. \& Wang, X. D. TNF-alpha induces two distinct caspase-8 activation pathways. Cell 133, 693-703 (2008).

11. Dickens, L. S. et al. A death effector domain chain DISC model reveals a crucial role for caspase-8 chain assembly in mediating apoptotic cell death. Mol. Cell 47, 291-305 (2012).

12. He, S. D. et al. Receptor interacting protein kinase-3 determines cellular necrotic response to TNF-alpha. Cell 137, 1100-1111 (2009).

13. Cai, Z. Y. et al. Plasma membrane translocation of trimerized MLKL protein is required for TNF-induced necroptosis (vol 16, pg 55, 2014). Nat. Cell Biol. 16 200-200 (2014).

14. Chen, X. et al. Translocation of mixed lineage kinase domain-like protein to plasma membrane leads to necrotic cell death. Cell Res. 24, 105-121 (2014).

15. Dondelinger, Y. et al. MLKL compromises plasma membrane integrity by binding to phosphatidylinositol phosphates. Cell Rep. 7, 971-981 (2014).

16. Su, L. J. et al. A plug release mechanism for membrane permeation by MLKL. Structure 22, 1489-1500 (2014).

17. Wang, H. Y. et al. Mixed lineage kinase domain-like protein MLKL causes necrotic membrane disruption upon phosphorylation by RIP3. Mol. Cell 54, 133-146 (2014).

18. Kelliher, M. A. et al. The death domain kinase RIP mediates the TNF-induced NF-kappa B signal. Immunity 8, 297-303 (1998)

19. Dowling, J. P., Nair, A. \& Zhang, J. A novel function of RIP1 in postnatal development and immune homeostasis by protecting against RIP3-dependent necroptosis and FADD-mediated apoptosis. Front. Cell Dev. Biol. 3, 12 (2015).

20. Kaiser, W. J. et al. RIP1 suppresses innate immune necrotic as well as apoptotic cell death during mammalian parturition. Proc. Natl Acad. Sci. USA 111, 7753-7758 (2014).

21. Dillon, C. P. et al. RIPK1 blocks early postnatal lethality mediated by caspase8 and RIPK3. Cell 157, 1189-1202 (2014).

22. Rickard, J. A. et al. RIPK1 regulates RIPK3-MLKL-driven systemic inflammation and emergency hematopoiesis. Cell 157, 1175-1188 (2014).

23. Takahashi, N. et al. RIPK1 ensures intestinal homeostasis by protecting the epithelium against apoptosis. Nature 513, 95-99 (2014).

24. Dannappel, M. et al. RIPK1 maintains epithelial homeostasis by inhibiting apoptosis and necroptosis. Nature 513, 90-94 (2014).

25. Zhang, H. B. et al. Functional complementation between FADD and RIP1 in embryos and lymphocytes. Nature 471, 373-+ (2011).

26. Newton, K. et al. Activity of protein kinase RIPK3 determines whether cells die by necroptosis or apoptosis. Science 343, 1357-1360 (2014).
27. Stanger, B. Z., Leder, P., Lee, T. H., Kim, E. \& Seed, B. Rip-a novel protein containing a death domain that interacts with Fas/Apo-1 (Cd95) in yeast and causes. Cell Death Cell 81, 513-523 (1995).

28. Christofferson, D. E., Li, Y. \& Yuan, J. Y. Control of life-or-death decisions by RIP1 kinase. Annu Rev. Physiol. 76, 129-150 (2014).

29. Degterev, A. et al. Identification of RIP1 kinase as a specific cellular target of necrostatins. Nat. Chem. Biol. 4, 313-321 (2008).

30. Dondelinger, Y. et al. NF-kappa B-independent role of IKK alpha/IKK beta in preventing RIPK1 kinase-dependent apoptotic and necroptotic cell death during TNF signaling. Mol. Cell 60, 63-76 (2015).

31. Geng J. F., et al. Regulation of RIPK1 activation by TAK1-mediated phosphorylation dictates apoptosis and necroptosis. Nat. Commun. 8, 359 (2017)

32. Polykratis, A. et al. Cutting edge: RIPK1 kinase inactive mice are viable and protected from TNF-induced necroptosis in vivo. J. Immunol. 193, 1539-1543 (2014).

33. Berger, S. B. et al. Cutting edge: RIP1 kinase activity is dispensable for normal development but is a key regulator of inflammation in SHARPIN-deficient mice. J. Immunol. 192, 5476-5480 (2014).

34. Li, J. X. et al. The RIP1/RIP3 necrosome forms a functional amyloid signaling complex required for programmed necrosis. Cell 150, 339-350 (2012)

35. Lin, J. et al. RIPK1 counteracts ZBP1-mediated necroptosis to inhibit inflammation. Nature 540, 124-128 (2016).

36. Newton, K. et al. RIPK1 inhibits ZBP1-driven necroptosis during development. Nature 540, 129-133 (2016).

37. Meng, H. Y. et al. Death-domain dimerization-mediated activation of RIPK controls necroptosis and RIPK1-dependent apoptosis. Proc. Natl Acad. Sci. USA 115, E2001-E2009 (2018).

38. O'Donnell, M. A., Legarda-Addison, D., Skountzos, P., Yeh, W. C. \& Ting, A. T. Ubiquitination of RIP1 regulates an NF-kappaB-independent cell-death switch in TNF signaling. Curr. Biol. 17, 418-424 (2007).

39. Tenev, T. et al. The ripoptosome, a signaling platform that assembles in response to genotoxic stress and loss of IAPs. Mol. Cell 43, 432-448 (2011).

40. Wertz, I. E. et al. Phosphorylation and linear ubiquitin direct A20 inhibition of inflammation. Nature 528, 370-375 (2015).

41. Zhang, X. X. et al. MLKL and FADD are critical for suppressing progressive lymphoproliferative disease and activating the NLRP3 inflammasome. Cell Rep. 16, 3247-3259 (2016).

42. Dillon, C. P. et al. Survival function of the FADD-CASPASE-8-cFLIP(L) complex. Cell Rep. 1, 401-407 (2012).

43. Alvarez-Diaz, S. et al. The pseudokinase MLKL and the kinase RIPK3 have distinct roles in autoimmune disease caused by loss of death-receptor-induced apoptosis. Immunity 45, 513-526 (2016).

44. Justus, S. J. \& Ting, A. T. Cloaked in ubiquitin, a killer hides in plain sight: the molecular regulation of RIPK1. Immunol. Rev. 266, 145-160 (2015).

45. Weinlich, R. \& Green, D. R. The two faces of receptor interacting protein kinase-1. Mol. Cell 56, 469-480 (2014).

46. Kelliher, M. A. et al. The death domain kinase RIP mediates the TNF-induced NF-kappaB signal. Immunity 8, 297-303 (1998)

47. Beg, A. A., Sha, W. C., Bronson, R. T., Ghosh, S. \& Baltimore, D. Embryonic lethality and liver degeneration in mice lacking the RelA component of NFkappa B. Nature 376, 167-170 (1995).

48. Li, Q., Van Antwerp, D., Mercurio, F., Lee, K. F. \& Verma, I. M. Severe liver degeneration in mice lacking the IkappaB kinase 2 gene. Science 284, 321-325 (1999).

49. Rudolph, D. et al. Severe liver degeneration and lack of NF-kappaB activation in NEMO/IKKgamma-deficient mice. Genes Dev. 14, 854-862 (2000).

50. Xu, C. et al. Embryonic lethality and host immunity of rela-deficient mice are mediated by both apoptosis and necroptosis. J. Immunol. 200, 271-285 (2018).

51. Vlantis, K. et al. NEMO prevents RIP kinase 1-mediated epithelial cell death and chronic intestinal inflammation by NF-kappaB-dependent and -independent functions. Immunity 44, 553-567 (2016).

52. Cuchet-Lourenco, D. et al. Biallelic RIPK1 mutations in humans cause severe immunodeficiency, arthritis, and intestinal inflammation. Science 361, 810-813 (2018)

53. Yang, J. et al. Type I interferons triggered through the toll-like receptor 3TRIF pathway control coxsackievirus A16 infection in young mice. J. Virol. 89 10860-10867 (2015)

\section{Acknowledgements}

We thank Xiaodong Wang (National Institute of Biological Sciences, Beijing, China) for providing Ripk $3^{-1-}$ mice, Jianke Zhang (Thomas Jefferson University, Philadelphia, PA, USA) for providing Fadd ${ }^{+/-}$mice, Feng Shao (National Institute of Biological Sciences, Beijing, China) for providing Tnfr1 $1^{-/-}$mice, and Qibin Leng (Institute Pasteur of Shanghai, Shanghai, China) for providing Ifnar ${ }^{-/-}$mice. We thank Junying Yuan for providing p-RIPK1(S166) antibody. We also thank Ronggui Hu (Shanghai Institute of Biochemistry and Cell Biology) and Hui Xiao (Institute Pasteur of Shanghai, Shanghai, China) for providing reagent and technical support. We thank Yu Sun (UCLA, Los 
Angeles, California) for valuable suggestions and comments on this study. This work was supported by grants from the National Key Research and Development Program of China (2016YFC1304900, 2016YFA0500100) and the National Natural Science Foundation of China $(31771537,31571426)$.

\section{Author contributions}

X.Z., Haiwei Z., and Haibing Z. conceived and designed the study; X.Z. and Haiwei Z. performed the experiments and analyzed data with assistance from C.X., X.L., M.L., W.P., and X.W.; B.Z. and Haikun W. analyzed data and provided technical support; D.L., Q.D., H.Y., and Hui W. provided essential reagents and intellectual input. X.Z., Haiwei Z., and Haibing Z. coordinated the project, interpreted results, and wrote the paper; Haibing $Z$. supervised the project.

\section{Additional information}

Supplementary Information accompanies this paper at https://doi.org/10.1038/s41467019-11839-w.

Competing interests: The authors declare no competing interests.

Reprints and permission information is available online at http://npg.nature.com/ reprintsandpermissions/
Peer review information: Nature Communications thanks the anonymous reviewers for their contribution to the peer review of this work. Peer reviewer reports are available.

Publisher's note: Springer Nature remains neutral with regard to jurisdictional claims in published maps and institutional affiliations.

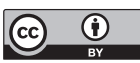

Open Access This article is licensed under a Creative Commons Attribution 4.0 International License, which permits use, sharing, adaptation, distribution and reproduction in any medium or format, as long as you give appropriate credit to the original author(s) and the source, provide a link to the Creative Commons license, and indicate if changes were made. The images or other third party material in this article are included in the article's Creative Commons license, unless indicated otherwise in a credit line to the material. If material is not included in the article's Creative Commons license and your intended use is not permitted by statutory regulation or exceeds the permitted use, you will need to obtain permission directly from the copyright holder. To view a copy of this license, visit http://creativecommons.org/ licenses/by/4.0/.

(c) The Author(s) 2019 